\title{
Uncovering an axion mechanism with the EDM portfolio
}

\author{
Jordy de Vries, ${ }^{1,2}$ Patrick Draper $\odot,{ }^{3}$ Kaori Fuyuto, ${ }^{4}$ Jonathan Kozaczuk, ${ }^{5}$ and Benjamin Lillard $\odot^{3}$ \\ ${ }^{1}$ Institute for Theoretical Physics Amsterdam and Delta Institute for Theoretical Physics, University of \\ Amsterdam, Science Park 904, 1098 XH Amsterdam, Netherlands \\ ${ }^{2}$ Nikhef, Theory Group, Science Park 105, 1098 XG Amsterdam, Netherlands \\ ${ }^{3}$ Department of Physics, University of Illinois, Urbana, Illinois 61801, USA \\ ${ }^{4}$ Theoretical Division, Los Alamos National Laboratory, Los Alamos, New Mexico 87545, USA \\ ${ }^{5}$ Department of Physics, University of California, San Diego, California 92093, USA
}

(Received 21 July 2021; accepted 19 August 2021; published 27 September 2021)

\begin{abstract}
Effective field theory arguments suggest that if beyond the standard model (BSM) sectors contain new sources of $C P$ violation that couple to $\mathrm{QCD}$, these sources will renormalize the $\theta$ term and frustrate ultraviolet solutions to the strong $C P$ problem. Simultaneously, they will generate distinctive patterns of low-energy electric dipole moments in hadronic, nuclear, atomic, and molecular systems. Observing such patterns thus provides evidence that strong $C P$ is solved by an infrared relaxation mechanism. We illustrate the renormalization of $\theta$ and the collections of electric dipole moments generated in several models of BSM physics, confirming effective field theory expectations, and demonstrate that measurements of ratios of electric dipole moments at planned experiments can provide valuable input on the resolution of the strong $C P$ problem.
\end{abstract}

DOI: 10.1103/PhysRevD.104.055039

\section{INTRODUCTION}

The universe violates parity $(P)$ and charge-parity $(C P)$ symmetries. In the standard model (SM), the weak interactions break $P$ and $C P$, while, beyond the standard model, new sources of $C P$ violation are required to generate the baryon asymmetry. For these reasons, it is a surprise that the strong interactions appear to be $P / C P$ symmetric. Even at the renormalizable level, the strong interactions contain a $P / C P$-violating $(\mathrm{CPV})$ phase $\bar{\theta}$. The limit on the neutron electric dipole moment (EDM), however, presently places a bound $\bar{\theta}<1.2 \times 10^{-10}[1,2]$. If $P$ and $C P$ are not symmetries of the Universe, why, then, does the strong force appear to conserve these quantum numbers to fantastic precision? Under renormalization, we might expect an $\mathrm{O}(1)$ $\bar{\theta}$ to be generated, even if for some reason it vanishes as an ultraviolet (UV) boundary condition.

It is a curious fact that this naïve renormalization group expectation is not reflected by the SM alone, even if it appears to be true in generic extensions of the SM. Radiative corrections to $\bar{\theta}$ in the SM start at high loop order [3] and are estimated to be well below the current experimental bound. This surprising property is the starting

Published by the American Physical Society under the terms of the Creative Commons Attribution 4.0 International license. Further distribution of this work must maintain attribution to the author(s) and the published article's title, journal citation, and DOI. Funded by SCOAP ${ }^{3}$. point for $\mathrm{UV}$ solutions to the strong $C P$ problem, including Nelson-Barr models based on spontaneous $C P$ violation, models based on spontaneous parity violation, and others [4-11]. If the UV Lagrangian preserves $P, C P$, or suitable generalizations of them, then $\bar{\theta}=0$ is a natural UV boundary condition. These symmetries must be broken spontaneously, and the trick is to sequester the order parameters sufficiently from QCD so that $\bar{\theta}$ is not regenerated when the spontaneous symmetry breaking sector is integrated out. This is a subtle model-building problem, because the fact that $\bar{\theta}$ is negligibly renormalized in the SM appears to be quite special, due to the limited flavor structures of the SM, and is not a property shared by most extensions of the SM [12-18]. Minimal left-right models, for example, preserve parity in the $\mathrm{UV}$, so that $\bar{\theta}=0$ is a natural boundary condition, but it is regenerated after electroweak symmetry breaking by new phases in the Higgs sector. However, various classes of UV solutions are known that might achieve the required sequestration, in which case the special radiative structure of the SM takes over and protects $\bar{\theta}$ into the infrared (IR).

Generically, one does not expect to observe any new sources of hadronic $C P$ violation if strong $C P$ is addressed by a UV mechanism. The sequestering required to protect $\bar{\theta}$ from spontaneous $P / C P$ breaking to several loop order also typically prevents any other sources of hadronic $C P$ violation-various dimension-six operators, in the language of the SM effective field theory (SMEFT) - from being generated at an observable level. This can be verified 
in explicit models for UV solutions. For example, the twoloop corrections to $\bar{\theta}$ in models of softly broken generalized parity typically dominate the pattern of EDMs [19]. More generally, the argument can be sharpened examining the divergence structure of SMEFT [18]. dimension-six CPV operators coupling to quarks or gluons give rise to one-loop quadratic divergences in $\bar{\theta}$, reflecting strong sensitivity of $\bar{\theta}$ to the CPV physics at the cutoff.

Contrapositively, UV models proposed to address strong $C P$ can be constrained by experimental evidence for new (non- $\bar{\theta}$ ) sources of hadronic $C P$ violation. The argument is one of naturalness: even if $\bar{\theta}$ is small in the UV, if other sources of hadronic $C P$ violation are present at an observable level, it is difficult to understand from an effective field theory point of view why $\bar{\theta}$ should be small in the IR. Thus, if new sources of strong-sector $C P$ violation are observed, axion solutions to the strong $C P$ problem-which largely relax the IR value of $\bar{\theta}$ regardless of its renormalization at intermediate scales and the presence of higher dimension operators-become essentially the only game in town.

This EFT argument gives low energy EDM experiments a unique and interesting window into the strong $C P$ problem. These experiments are the most sensitive probes of new sources of flavor-diagonal CPV beyond the SM. A complication, however, is that a small bare $\bar{\theta}$ itself generates a pattern of hadronic EDMs. Therefore, correlated measurements are required to distinguish a pure $\bar{\theta}$ scenario from one with distinct new CPV sources. Given a sufficient suite of hadronic EDM measurements, if nonzero EDMs are observed, it is possible to disentangle the various contributions from a small nonzero $\bar{\theta}$ and other CPV sources. If the latter are present, the argument above can be applied.

In this work we illustrate how this EFT argument operates by studying a few concrete UV extensions of the SM. These models introduce new $C P$-violating interactions for quarks and gluons somewhere above the weak scale. We show that these interactions renormalize $\bar{\theta}$ at tree level or one loop, necessitating an IR solution to the strong $C P$ problem. Furthermore, we show that the models generate distinctive correlated patterns of EDMs distinguishable from the pattern generated by $\bar{\theta}$ alone.

This paper is organized as follows. In Sec. II we discuss the renormalization of $\bar{\theta}$ in the SM and beyond. We recap how $\bar{\theta}$ is corrected in SMEFT and introduce three beyond the standard model (BSM) models with additional sources of $C P$ violation that explicitly verify the EFT expectations. In Sec. III we discuss the low-energy $C P$-odd interactions that are induced in the BSM models and the resulting EDMs of hadrons, nuclei, atoms, and molecules. In Sec. IV we demonstrate how the pattern of EDMs of different systems can separate pure $-\bar{\theta}$ scenarios (which can arise either in IR or UV solutions to the strong $C P$ problem) from models where higher-dimensional flavor-diagonal $C P$-odd operators are relevant and an IR solution is needed. We conclude in Sec. V.

\section{CORRECTIONS TO $\bar{\theta}$}

In the $\mathrm{SM}$, the strong $C P$ phase that is invariant under anomalous chiral field redefinitions is

$$
\bar{\theta}=\theta+\arg \operatorname{det} y_{u} y_{d},
$$

where $y_{u, d}$ are the up- and down-type Yukawa matrices. In BSM models, there can be additional terms in the invariant strong $C P$ phase. There can also be new invariant phases that radiatively correct $\bar{\theta}$. We will see examples of both below.

In perturbation theory, a radiative correction to $\arg \operatorname{det} y$ shifts $\bar{\theta}$ at one loop by

$$
\begin{aligned}
\Delta \bar{\theta} & =\arg \operatorname{det}(y+\delta y)-\arg \operatorname{det}=\arg \operatorname{det}\left(1+y^{-1} \delta y\right) \\
& \approx \operatorname{Im} \operatorname{tr}\left(y^{-1} \delta y\right),
\end{aligned}
$$

where $y \equiv y_{u} y_{d}$.

\section{A. Corrections to $\overline{\boldsymbol{\theta}}$ in SMEFT}

In SMEFT, there are quadratic divergences in the one-loop radiative contribution to $\bar{\theta}$ arising from higher-dimensional $C P$-violating operators. These terms can be used to estimate the correction to $\bar{\theta}$ in a systematic computation, as long as the Wilsonian momentum cutoff is taken sufficiently below the scale at which the SMEFT operators become strongly coupled. Typically, this is a conservative estimate. More generally, the quadratic divergences can be taken simply as an indicator that models which generate hadronic $C P$ violating SMEFT operators typically also have sizeable new threshold corrections to $\bar{\theta}$. The explicit calculation was performed in Ref. [18] and here we just list the result.

We define the dimension-six operators through

$$
\mathcal{L}_{\mathrm{SMEFT}}=\mathcal{L}_{\mathrm{SM}}+\frac{1}{\Lambda^{2}} \sum_{i} c_{i} \mathcal{O}_{i}
$$

in terms of the operators in Table I. The one-loop quadratic divergence in $\bar{\theta}$ from dimension-six $C P$-violating SMEFT operators is [18]

TABLE I. Dimension-six SMEFT operators in the basis of Ref. [20], which contribute to the renormalization of $\bar{\theta}$ at one loop.

\begin{tabular}{lccc}
\hline \hline $\mathcal{O}_{u H}$ & $H^{\dagger} H \overline{Q_{L i}} \tilde{H} u_{R j}$ & $\mathcal{O}_{d H}$ & $H^{\dagger} H \overline{Q_{L i}} H d_{R j}$ \\
$\mathcal{O}_{d G}$ & $\overline{Q_{L i}} \sigma^{\mu \nu} T^{a} d_{R j} H G_{\mu \nu}^{a}$ & $\mathcal{O}_{d W}$ & $\overline{Q_{L i}} \sigma^{\mu \nu} d_{R j} \tau^{a} H W_{\mu \nu}^{a}$ \\
$\mathcal{O}_{d B}$ & $\overline{Q_{L i}} \sigma^{\mu \nu} d_{R j} H B_{\mu \nu}$ & $\mathcal{O}_{u G}$ & $\overline{Q_{L i}} \sigma^{\mu \nu} T^{a} u_{R j} \tilde{H} G_{\mu \nu}^{a}$ \\
$\mathcal{O}_{u W}$ & $\overline{Q_{L i}} \sigma^{\mu \nu} u_{R j} \tau^{a} \tilde{H} W_{\mu \nu}^{a}$ & $\mathcal{O}_{u B}$ & $\overline{Q_{L i}} \sigma^{\mu \nu} u_{R j} \tilde{H} B_{\mu \nu}$ \\
$\mathcal{O}_{H u d}$ & $i \tilde{H}^{\dagger} D_{\mu} H \overline{u_{R i}} \gamma^{\mu} d_{R j}$ & $\mathcal{O}_{q u q d(1)}$ & $\epsilon^{e f} \overline{Q_{L i}^{e}} u_{R j} \overline{Q_{L k}^{f}} d_{R l}$ \\
$\mathcal{O}_{q u q d(8)}$ & $\epsilon^{e f} \overline{Q_{L i}^{e}} T^{a} u_{R j} \overline{Q_{L k}^{f}} T^{a} d_{R l}$ & $\mathcal{O}_{\text {lequ(1) }}$ & $\epsilon^{e f} \overline{L_{L i}^{e}} e_{R j} \overline{Q_{L k}^{f}} u_{R l}$ \\
$\mathcal{O}_{\text {ledq }}$ & $\overline{L_{L i}} e_{R j} \overline{d_{R k} Q_{L l}}$ & $\mathcal{O}_{H \tilde{G}}$ & $H^{\dagger} H G_{\mu \nu}^{a} \tilde{G}^{a \mu \nu}$ \\
$\mathcal{O}_{\tilde{G}}$ & $f^{a b} G_{\nu}^{a \mu} G_{\rho}^{b \nu} \tilde{G}_{\mu}^{c \rho}$ & & \\
\hline \hline
\end{tabular}




$$
\begin{aligned}
16 \pi^{2} \delta \bar{\theta} \sim & 16 \pi^{2}\left(\frac{2}{g_{s}^{2}} c_{H \tilde{G}}-\frac{9}{2 g_{s}} c_{\tilde{G}}\right) \\
& +\operatorname{Im} \operatorname{Tr}\left[Y_{d}^{-1}\left(3 c_{d H}+g^{\prime} c_{d B}-18 g c_{d W}-16 g_{s} c_{d G}\right)\right] \\
& +\operatorname{Im} \operatorname{Tr}\left[Y_{u}^{-1}\left(3 c_{u H}-5 g^{\prime} c_{u B}-18 g c_{u W}-16 g_{s} c_{u G}\right)\right] \\
& +\operatorname{Im} \operatorname{Tr}\left[\left(Y_{d}^{-1} Y_{u}+Y_{d}^{\dagger}\left(Y_{u}^{\dagger}\right)^{-1}\right) c_{H u d}\right] \\
& +\operatorname{Im}\left[2 c_{l e q u(1)}^{m n i j} Y_{e}^{\dagger n m}\left(Y_{u}^{-1}\right)^{j i}-2 c_{l e d q}^{* m n i j} Y_{e}^{m n}\left(Y_{d}^{-1}\right)^{i j}\right] \\
& +\operatorname{Im}\left[\left(6 c_{q u q d(1)}^{m n i j}+c_{q u q d(1)}^{i n m j}+\frac{4}{3} c_{q u q d(8)}^{i n m j}\right)\left(Y_{u}^{\dagger n m}\left(Y_{d}^{-1}\right)^{j i}+Y_{d}^{\dagger j i}\left(Y_{u}^{-1}\right)^{n m}\right)\right]
\end{aligned}
$$

Absent an infrared relaxation of $\bar{\theta}$, e.g., by the PecceiQuinn mechanism, naturalness requires $|\delta \bar{\theta}| \lesssim 10^{-10}$, implying a stringent bound on the combination of Wilson coefficients in Eq. (4).

Now we consider specific UV completions of the SM where the threshold corrections to $\bar{\theta}$ and the predictions for numerous EDM observables can be computed explicitly. We confirm the EFT expectation that whenever a dimension-six operator is introduced that appears in Eq. (4), the dominant source of $C P$ violation should be a $\bar{\theta}$ term much larger than the present bound, unless it is relaxed by a Peccei-Quinn mechanism.

Axion models [21-28] provide the standard IR solution to the strong $C P$ problem. The QCD axion nonlinearly realizes an approximate chiral $U(1)_{\mathrm{PQ}}$ symmetry, with decay constant $f_{a} \gtrsim 10^{9} \mathrm{GeV}$. The axion $a$ modifies the $C P$-odd Lagrangian term for the gluons, $\mathcal{L} \rightarrow\left(\bar{\theta}+a / f_{a}\right) G \tilde{G}$, so that the effective value of $\bar{\theta}(a)$ is modified by the axion vacuum expectation value (VEV). Nonperturbative QCD effects explicitly break $U(1)_{\mathrm{PQ}}$ and generate a periodic potential for the axion that is minimized at $\bar{\theta}+a / f_{a}=0$, thus setting the effective $C P$-violating phase to zero in the vacuum of the axion model, $\langle\bar{\theta}(a)\rangle \equiv \bar{\theta}+\langle a\rangle / f_{a}=0$. Additional sources of $U(1)_{\mathrm{PQ}}$ violation can shift the minimum of the axion potential to a nonzero value of $\langle\bar{\theta}(a)\rangle$. To ensure a sufficiently small $|\bar{\theta}|<10^{-10}$, the $U(1)_{\mathrm{PQ}}$ global symmetry must be nearly exact, broken only by QCD effects or by very highdimension irrelevant operators (often constrained to be higher than dimension ten). This is referred to as the axion quality problem. A handful of models are known to protect $U(1)_{\mathrm{PQ}}$ to a sufficient degree without fine-tuning the coupling constants, often as an "accident" of some other structure in the ultraviolet theory [29-41].

\section{B. Case I: Scalar leptoquark models}

We start our analysis in a very simple SM extension, a model of scalar leptoquarks (LQs). Recently these models have been investigated extensively due to anomalies in $b \rightarrow$ sll transitions and the muon anomalous magnetic moment. We consider here the $R_{2}$ scalar LQ [42] that carries SM gauge quantum numbers (3, 2,7/6). Apart from interactions among LQs and LQ-Higgs interactions that conserve $C P$, there are potentially $C P$-violating interactions between LQs and the SM fermions. We focus on couplings between quarks and charged leptons given by

$$
\mathcal{L}_{Y}^{\left(R_{2}\right)}=R_{2}^{I}\left(\bar{u}_{R} x_{R L} \epsilon_{I J} L^{J}+\bar{Q}^{I} x_{L R}^{\dagger} e_{R}\right)+\text { H.c., }
$$

where $x_{R L, L R}$ are $3 \times 3$ flavor matrices and $I, J$ denote $S U(2)$ indices. The presence of two interactions coupling to both left- and right-handed quarks and leptons leads to a rich EDM phenomenology [43,44]. The LQ doublet describes two states with charges $(5 / 3) e$ and $(2 / 3) e$ and we consider a common mass for these states $m_{R_{2}}$.

The relative phase in the LQ couplings lead to a threshold correction to $\bar{\theta}$. That is, even if $\bar{\theta}=0$, it is renormalized at one loop by phases in the $x$ couplings. Closing the lepton lines gives the following UV-divergent contribution

$$
\Delta \bar{\theta}=\frac{c}{8 \pi^{2}} \operatorname{Im} \operatorname{Tr}\left[y_{u}^{-1}\left(x_{R L} y_{e}^{\dagger} x_{L R}\right)\right] \log \left(\frac{\Lambda}{m_{R_{2}}}\right),
$$

where $\Lambda$ is a momentum cutoff and $c$ is an order one constant. We see the appearance of a second reparametrization-invariant phase,

$-\arg \operatorname{det} y_{u}-\arg \operatorname{det} y_{e}+\arg \operatorname{det} x_{R L}+\arg \operatorname{det} x_{L R}$.

While this contribution is suppressed by the lepton Yukawa, it can be enhanced by $1 / y_{u}$ for first-generation quarks. The same couplings also give rise to a correction to the electron mass

$$
\Delta m_{e} \sim \frac{1}{8 \pi^{2}}\left(x_{R L}^{e q} m_{q} x_{L R}^{q e}\right) \log \left(\frac{\Lambda}{m_{R_{2}}}\right),
$$

and on naturalness grounds we require $\Delta m_{e} \lesssim m_{e}$. This says, for example, that 


$$
x_{R L}^{e t} x_{L R}^{t e} \log \left(\Lambda / m_{R_{2}}\right) \lesssim 10^{-4}
$$

However, it places essentially no constraint on firstgeneration couplings.

The phenomenology of EDMs in leptoquark models was discussed in detail in Refs. [43,44]. The LQ-fermion interactions lead to dimension-six operators below the scale of the LQ masses. Tree-level diagrams induce $[45,46]$

$$
\begin{aligned}
\mathcal{L}= & \frac{c_{\text {lequ }(1)}^{a b c d}}{\Lambda^{2}}\left(\bar{L}_{a}^{I} e_{R_{b}}\right) \epsilon_{I J}\left(\bar{Q}_{c}^{J} u_{R_{d}}\right) \\
& +\frac{c_{\text {lequ }(3)}^{a b c d}}{\Lambda^{2}}\left(\bar{L}_{a}^{I} \sigma^{\mu \nu} e_{R_{b}}\right) \epsilon_{I J}\left(\bar{Q}_{c}^{J} \sigma_{\mu \nu} u_{R_{d}}\right),
\end{aligned}
$$

where

$$
\frac{c_{l e q u(1)}^{a b c d}}{\Lambda^{2}}=4 \frac{c_{l e q u(3)}^{a b c d}}{\Lambda^{2}}=\frac{\left(x_{L R}\right)^{b c}\left(x_{R L}\right)^{d a}}{2 m_{R_{2}}^{2}} .
$$

Note that closing the lepton lines into a loop, the first operator contributes to the renormalization of $\bar{\theta}$ in SMEFT, cf. Eq. (4), which correctly matches the qualitative structure of the correction in the full LQ model, Eq. (6). In this model we have a direct correspondence between the EFT and full theory computations of the correction to $\bar{\theta}$. In the other models we consider, the SMEFT estimates will be conservative, with more important corrections to $\bar{\theta}$ arising already at tree level in the UV theory. The second operator also contributes to $\bar{\theta}$ but only at two-loop order. This is reflected by the mixing of $c_{\text {lequ(1) }}^{a b c d}$ and $c_{\text {lequ(3) }}^{a b c d}$ at one-loop order in QCD.

At the threshold of the top and charm quark, the fourfermion operators induce dimension-seven $C P$-violating lepton-gluon operators. We only consider the electron interactions

$\mathcal{L}_{e G}=C_{e G} \alpha_{s} \bar{e} i \gamma_{5} e G_{\mu \nu}^{a} G^{a \mu \nu}+C_{e \tilde{G}} \frac{\alpha_{s}}{2} \bar{e} e G_{\mu \nu}^{a} G_{\alpha \beta}^{a} \epsilon^{\alpha \beta \mu \nu}$,

where

$$
C_{e G}=-\frac{2}{3} C_{e \tilde{G}}=\sum_{q=c, t} \frac{1}{24 \pi} \frac{\operatorname{Im} c_{l e q u(1)}^{e e q q}}{\Lambda^{2} m_{q}} .
$$

Electric dipole moments of quarks and leptons and chromoelectric dipole moment of quarks are induced at the one-loop level. Focusing on first-generation quarks and leptons we define the operators ${ }^{1}$

\footnotetext{
${ }^{1}$ The dipole operators can be related to the coefficients in Eq. (4). For instance, $d_{u}=\left(e \sqrt{2} v / \Lambda^{2}\right) \operatorname{Im}\left(c_{u B}^{11} / g^{\prime}+c_{u W}^{11} / g\right)$ and $\tilde{d}_{u}=-\left(\sqrt{2} v / \Lambda^{2}\right) \operatorname{Im}\left(c_{u G}^{11} / g_{s}\right)$. The electron EDM does not appear in Eq. (4) as it only renormalizes $\bar{\theta}$ at the three-loop level.
}

$$
\begin{aligned}
\mathcal{L}_{\text {dipole }}= & -\frac{d_{e}}{2} \bar{e} \sigma^{\mu \nu} i \gamma^{5} e F_{\mu \nu}-\frac{d_{u}}{2} \bar{u} \sigma^{\mu \nu} i \gamma^{5} u F_{\mu \nu} \\
& -\frac{g_{s} \tilde{d}_{u}}{2} \bar{u} \sigma^{\mu \nu} i \gamma^{5} t^{a} u G_{\mu \nu}^{a}
\end{aligned}
$$

where the one-loop expressions are given by

$$
\begin{aligned}
& d_{e}=\frac{e}{(4 \pi)^{2}} \sum_{q=u, c, t} \frac{N_{c} m_{q}}{6} \operatorname{Im}\left[\frac{\left(x_{L R}\right)^{e q}\left(x_{R L}\right)^{q e}}{m_{R_{2}}^{2}}\right]+\mathcal{O}\left(\frac{m_{q}^{2}}{m_{R_{2}}^{2}}\right), \\
& d_{u}=-\frac{e}{(4 \pi)^{2}} \sum_{l=e, \mu, \tau} \frac{2 m_{l}}{3} \operatorname{Im}\left[\frac{\left(x_{L R}\right)^{l u}\left(x_{R L}\right)^{u l}}{m_{R_{2}}^{2}}\right]+\mathcal{O}\left(\frac{m_{l}^{2}}{m_{R_{2}}^{2}}\right), \\
& \tilde{d}_{u}=-\frac{1}{(4 \pi)^{2}} \sum_{l=e, \mu, \tau} \frac{m_{l}}{2} \operatorname{Im}\left[\frac{\left(x_{L R}\right)^{l u}\left(x_{R L}\right)^{u l}}{m_{R_{2}}^{2}}\right]+\mathcal{O}\left(\frac{m_{l}^{2}}{m_{R_{2}}^{2}}\right) .
\end{aligned}
$$

In particular the electron EDM can get large contributions from internal top quarks.

If we focus on LQ couplings for first-generation quarks and leptons only, the largest contributions are to $C P$-odd fourfermion operators while the dipole operators are loop suppressed. For electron-top-LQ interactions this is no longer the case, as the electron-gluon operators also arise at one loop and the electron EDM is enhanced by $m_{t} / m_{e}$. The resulting EDM phenomenology will be discussed in Sec. III.

\section{Case II: The minimal supersymmetric standard model}

In the minimal supersymmetric standard model (MSSM), the invariant strong $C P$ phase is

$$
\bar{\theta}=\theta+\arg \operatorname{det} y_{u} y_{d}+3 \arg m_{\tilde{g}}+3 \arg v_{u} v_{d} .
$$

It receives new tree-level contributions from the last two terms. It also receives radiative corrections, for example, from one-loop contributions to the quark masses or the gluino mass that are sensitive to phases in the $A$ terms and the $\mu$ term [12]. Rather than studying the full set of oneloop corrections to $\bar{\theta}$, we restrict our attention to those contributions that are proportional to $\alpha_{s}$, as this is sufficient to generate a nontrivial pattern of electric dipole moments.

At one-loop order in $\alpha_{s}$, the Yukawa couplings $y_{u, d}$ receive two types of corrections. The first type is proportional to $A_{u, d}$, and in a highly simplified limit the correction to $\bar{\theta}$ is

$$
\Delta \bar{\theta} \approx \frac{\alpha_{s}}{3 \pi} \frac{1}{m_{\text {soft }}^{2}} \operatorname{Im} \operatorname{Tr}\left[y_{u}^{-1}\left(m_{\tilde{g}}^{\dagger} A_{u}\right)\right]+(u \leftrightarrow d) .
$$

Here we work in a flavor basis where the gluino-quarksquark couplings are real and proportional to the identity in flavor space, and for simplicity we take the limits where the squarks are degenerate at $m_{\text {soft }}$, the gluino has similar mass 
$\left|m_{\tilde{g}}\right|^{2} \approx m_{\text {soft }}^{2}$, and the $A$ terms are treated in the insertion approximation. We see that if the gluino mass is real, and in the limit of exact proportionality $A_{f} \propto y_{f}$, the corrections to $\bar{\theta}$ vanish. However, if we allow for deviations from exact proportionality, $\Delta \bar{\theta}$ is in general nonzero because of phases in the Yukawas, even if the gluino phase vanishes at tree level. Furthermore, if we allow deviations from exact degeneracy of the squark masses, the trace above will include insertions of flavor-changing squark soft masses $\delta \tilde{m}_{f f^{\prime}}^{2}$, e.g.:

$$
(\Delta \bar{\theta})_{A} \simeq \frac{\alpha_{s}}{18 \pi} \frac{1}{m_{\mathrm{soft}}^{6}} \operatorname{Im} \operatorname{Tr}\left[y_{u}^{-1} m_{\tilde{g}}^{\dagger}\left(\delta \tilde{m}^{2}\right) A_{u}\left(\delta \tilde{m}^{2}\right)\right]+(u \leftrightarrow d) .
$$

This correction can shift $\bar{\theta}$ even in the limit of exact $A$-termYukawa proportionality.

A second contribution to the quark masses comes from the $\mu$ term in the superpotential, which induces cubic scalar interactions $\mathcal{L} \supset \mu^{\star}\left[\tilde{u}_{R} y_{u} \tilde{u}_{L} H_{d}^{0 \star}+(d \leftrightarrow u)\right]+$ H.c. With the $H_{u, d}^{0} \rightarrow v_{u, d} / \sqrt{2}$ VEV insertion,

$$
\delta m_{u}^{i j} \approx-\frac{\alpha_{s}}{3 \pi} \mu^{\star} m_{g}^{\star} \frac{v_{d}^{\star}}{\sqrt{2}} y_{u}^{i j} \frac{1}{m_{\text {soft }}^{2}},
$$

where again we simplify the loop integrals by setting the squark and gluino propagator masses to a universal $m_{\text {soft }}$. An analogous expression for $\delta m_{d}^{i j}$ is found by applying $(u \leftrightarrow d)$ above. Each correction $\delta y_{q}$ is strictly proportional to $y_{q}$, so the new contribution to $\bar{\theta}$ is

$$
\begin{aligned}
(\Delta \bar{\theta})_{\mu} & \simeq \operatorname{Im} \operatorname{Tr}\left(y_{u}^{-1} \delta y_{u}+y_{d}^{-1} \delta y_{d}\right) \\
& =-\frac{\alpha_{s}}{\pi} \operatorname{Im}\left[\left(\frac{v_{d}^{\star}}{v_{u}}+\frac{v_{u}^{\star}}{v_{d}}\right) \frac{\mu^{\star} m_{g}^{\star}}{m_{s}^{2}}\right] .
\end{aligned}
$$

Note that in the limit of large $\tan \beta \equiv\left|v_{u} / v_{d}\right|$, the assumption $\delta y_{u} \ll y_{u}$ underpinning the Eq. (2) approximation can become invalid if $\mu \tan \beta \alpha_{s} / \pi \gtrsim \mathcal{O}\left(m_{s}\right)$, so a more careful analysis is required in that regime [47].

From the gluino mass correction, $\Delta \bar{\theta} \approx 3 \operatorname{Im}\left(m_{g}^{-1} \delta m_{g}\right)$, with similar assumptions for the particle masses and couplings, we obtain

$$
(\Delta \bar{\theta})_{v^{2}}=-\frac{3 \alpha_{s}}{16 \pi} \frac{v_{u}^{2}}{m_{\mathrm{soft}}^{2}} \operatorname{Im}\left(m_{\tilde{g}}^{-1} \operatorname{Tr}\left(A_{u} y_{u}^{\dagger}\right)\right)+(u \leftrightarrow d) .
$$

In each case, we see the appearance of additional phases that can correct $\bar{\theta}$. The relevant reparametrization-invariant phases can be read off directly from each of the corrections (and in cases involving VEVs note that the soft supersymmetry (SUSY)-breaking $B$ term transforms like $v_{u}^{*} v_{d}^{*}$ ). If there is a large hierarchy between the weak scale and the SUSY-breaking scale, the correction to the gluino mass phase can be suppressed below $10^{-10}$; however, this suppression does not affect the correction to the quark mass phases. To remove all the large sources of $\Delta \bar{\theta}$ in the MSSM, additional strong assumptions must be made. For example, in soft supersymmetry breaking universality, the Higgs VEVs and gaugino masses are taken to be real, and the $A$ terms are taken to be exactly proportional to the Yukawa matrices.

In the MSSM with $R$-parity conservation, there are no tree-level contributions to SMEFT operators, but the dipole

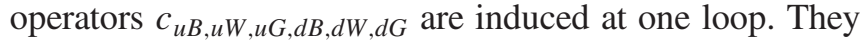
appear in Eq. (4); closing the gauge-boson loop corresponds to a two-loop contribution to $\bar{\theta}$. In the full model there are larger, one-loop corrections to $\bar{\theta}$ from diagrams with the gauge boson removed entirely, not to mention treelevel contributions. So in this case the SMEFT estimate is likely conservative. Nevertheless, it is convenient as a diagnostic tool: the appearance of any dimension-six operators appearing in Eq. (4), which can be verified by low-energy experiments as discussed in detail below, suggests large corrections to $\bar{\theta}$.

The quark EDMs and chromo-EDMs (CEDMs) $\left(d_{q}\right.$ and $\tilde{d}_{q}$, respectively) are defined as in Eq. (14)

$$
\mathcal{L}=-\frac{d_{q}}{2} \bar{q} \sigma^{\mu \nu} i \gamma^{5} q F_{\mu \nu}-\frac{g_{s} \tilde{d}_{q}}{2} \bar{q} \sigma^{\mu \nu} i \gamma^{5} t^{a} q G_{\mu \nu},
$$

for $q=\{u, d\}$. Complex phases in the gluino mass and in the fermion-gluino interaction generate an EDM and CEDM at one-loop order, proportional to $\alpha_{s}$. For the EDM, the relevant diagram features a virtual gluino and squark, $q \rightarrow\left(\tilde{g}+\tilde{q}^{\prime}\right) \rightarrow q$, with a photon coupled to the virtual squark. Here the deviations from exact degeneracy in the squark masses become important, so we will treat them with a degree of generality, including off-diagonal (mass) $)^{2}$ couplings in the flavor basis. In the absence of any generation-changing masses, the first-generation up and down squarks (in the flavor basis) have mass matrices given approximately by [48]

$$
\begin{aligned}
M_{\tilde{u}}^{2} & \simeq\left(\begin{array}{cc}
M_{\tilde{Q}}^{2}+\mathcal{O}\left(m_{Z}^{2} \cos 2 \beta\right) & m_{u}\left(A_{u}^{\star}-\mu \cot \beta\right) \\
m_{u}\left(A_{u}-\mu^{\star} \cot \beta\right) & M_{\tilde{U}}^{2}+\mathcal{O}\left(m_{Z}^{2} \cos 2 \beta\right)
\end{array}\right) \\
& \approx m_{\text {soft }}^{2}\left(\begin{array}{cc}
1 & \epsilon_{u}^{\star} \\
\epsilon_{u} & 1+\Delta_{u}
\end{array}\right), \\
M_{\tilde{d}}^{2} & \simeq\left(\begin{array}{cc}
M_{\tilde{Q}}^{2}+\mathcal{O}\left(m_{Z}^{2} \cos 2 \beta\right) & m_{d}\left(A_{d}^{\star}-\mu \tan \beta\right) \\
m_{d}\left(A_{d}-\mu^{\star} \tan \beta\right) & M_{\tilde{D}}^{2}+\mathcal{O}\left(m_{Z}^{2} \cos 2 \beta\right)
\end{array}\right) \\
& \approx m_{\mathrm{soft}}^{2}\left(\begin{array}{cc}
1 & \epsilon_{d}^{\star} \\
\epsilon_{d} & 1+\Delta_{d}
\end{array}\right),
\end{aligned}
$$

where the $A_{u, d}$ above refer to the $(1,1)$ components of the respective $3 \times 3$ matrices. Above, we parametrize the mass 
matrices with a real $\Delta_{q}$ and complex $\epsilon_{q}$ for each $q=u, d$. Ignoring the $\mathcal{O}\left(m_{Z}^{2}\right)$ diagonal entries, we take the $(1,1)$ (i.e., left-left) components of the two matrices to be approximately equal to $m_{\text {soft }}^{2} \approx M_{\tilde{Q}}^{2}$.

In the approximately degenerate limit assumed for the squark masses in our calculation of the one-loop corrections to $\Delta \bar{\theta},\left|\Delta_{q}\right| \ll \mathcal{O}(1)$. Even with exactly degenerate soft SUSY-breaking masses $M_{\tilde{Q}}^{2}=M_{\tilde{U}}^{2}=M_{\tilde{D}}^{2}$, however, the $\mathcal{O}\left(m_{Z}^{2}\right)$ diagonal terms from electroweak symmetry breaking introduce some degree of mass splitting. Absent any fine cancellations between the electroweak corrections and the soft masses, we anticipate a lower limit on the magnitude of $\Delta,\left|\Delta_{q}\right| \gtrsim \mathcal{O}\left(\cos 2 \beta m_{Z}^{2} / m_{\text {soft }}^{2}\right)$. The offdiagonal $\epsilon_{q}$, on the other hand, are suppressed by factors of $m_{q} / m_{\text {soft }}$, so we may assume $|\epsilon| \ll|\Delta|$ for the firstgeneration quarks.

Following $[47,48]$, the contribution from the gluino loop diagram to the quark EDM $d_{q}$ is given by

$$
\frac{d_{q}}{e}=-\frac{2 \alpha_{s}}{3 \pi} \sum_{j=1,2} \operatorname{Im}\left(U_{q 2 j} U_{q 1 j}^{\star} e^{-i \phi_{3}}\right) \frac{\left|m_{\tilde{g}}\right|}{M_{\tilde{q}_{j}}^{2}} Q_{\tilde{q}} B\left(\frac{\left|m_{\tilde{g}}\right|^{2}}{M_{\tilde{q}_{j}}^{2}}\right),
$$

where $\phi_{3}=\arg m_{\tilde{g}}$ is the phase of the gluino mass; the $U_{q}$ diagonalize the mass matrices via $U_{q}^{\dagger} M_{\tilde{q}}^{2} U_{q}=$ $\operatorname{diag}\left(M_{\tilde{q}_{1}}^{2}, M_{\tilde{q}_{2}}^{2}\right)$, with mass eigenvalues $M_{\tilde{q}_{j}}^{2} ; Q_{\tilde{q}}$ is the squark electric charge; and where

$$
B(r)=\frac{1+r+\frac{2 r}{1-r} \ln r}{2(1-r)^{2}}, \quad B(1)=\frac{1}{6} .
$$

Expanding to linear order in $\epsilon / \Delta$, we find $\left(U_{q}\right)_{11}=$ $\left(U_{q}\right)_{22} \simeq 1$, and $\left(U_{q}\right)_{12}=-\left(U_{q}\right)_{21}^{\star}=\epsilon^{\star} / \Delta$. Similarly, the mass eigenvalues are $M_{\tilde{q}_{1}}^{2} \simeq m_{\text {soft }}^{2}\left(1+\mathcal{O}\left(\epsilon^{2}\right)\right)$ and $M_{\tilde{q}_{2}}^{2} \simeq m_{\text {soft }}^{2}\left(1+\Delta+\mathcal{O}\left(\epsilon^{2}\right)\right)$. Specializing to the $\left|m_{\tilde{g}}^{2}\right| \approx$ $m_{\text {soft }}^{2}$ case, we find

$$
\begin{gathered}
\frac{d_{q}}{e} \approx-Q_{q} \frac{\alpha_{s}}{4 \pi} \frac{\left|\epsilon_{q}\right|}{m_{\text {soft }}} \sin \left(\phi_{3}-\arg \epsilon_{q}\right) \times F(\Delta), \\
F(\Delta) \equiv \frac{4}{9 \Delta^{4}}\left[\Delta^{3}-3 \Delta^{2}-6 \Delta+6(1+\Delta) \log (1+\Delta)\right] .
\end{gathered}
$$

In the limit of degenerate squark masses, $\Delta \rightarrow 0$, the function $F$ approaches a constant, $F(0)=2 / 9$. Recalling that the typical size of $\epsilon$ is $|\epsilon| \sim m_{q} / m_{\text {soft }}$, the overall contribution to the $q=u, d$ dipole moment scales as $m_{q} / m_{\text {soft }}^{2}$.

At one-loop order, the quark chromo-EDM is proportional to the same $\operatorname{Im}\left(e^{i \phi_{3}} \epsilon^{\star}\right)$ combination of phases, with the same dependence on $U_{q}$; the only major difference is the existence of a second type of diagram, where the external gluon couples to the virtual gluino rather than the squark, so that the gluino loop diagram induces a CEDM of

$$
\tilde{d}_{q} \approx \frac{\alpha_{s}}{4 \pi} \frac{\left|\epsilon_{q}\right|}{m_{\mathrm{soft}}} \sin \left(\phi_{3}-\arg \epsilon_{q}\right) \times G(\Delta),
$$

where

$$
\begin{aligned}
G(\Delta)= & \frac{1}{18 \Delta^{4}}\left[19 \Delta^{3}+78 \Delta^{2}+48 \Delta\right. \\
& -6(1+\Delta)(8+9 \Delta) \log (1+\Delta)] .
\end{aligned}
$$

Here we have again specialized to $m_{\tilde{g}}^{2} \approx m_{\text {soft }}^{2}$ in order to express the result simply in terms of $\Delta$. In the limit $\Delta \rightarrow 0$, $G(0)=5 / 18$ remains finite.

By turning on additional phases in the electroweak chargino sector, the EDMs and CEDMs receive further contributions, but as these are proportional to $g^{2}$ or $g^{\prime 2}$, they can be considered small compared to the $\alpha_{s}$ corrections listed above unless the gluino phase is tuned so that $\operatorname{Im}\left(e^{i \phi_{3}} \epsilon_{q}^{\star}\right)$ is small.

\section{Case III: The $P$-symmetric minimal left-right symmetric model}

Left-right symmetric models have an extended gauge symmetry $S U(3)_{c} \times S U(2)_{L} \times S U(2)_{R} \times U(1)_{B-L}$ [49-51]. The left- and right-handed fermions are fundamental representations of the $S U(2)$ groups, and right-handed neutrinos appear automatically. The minimal left-right symmetric model (MLRSM) includes a minimal scalar sector containing one scalar bidoublet and two triplets [52]. Vacuum expectation values of these scalar fields then break the extended gauge symmetry to the SM group at some highenergy scale. This leads to massive right-handed electroweak gauge and scalar bosons with masses above a few $\mathrm{TeV}$ to avoid phenomenological constraints. Full details of the model can be found in many places in the literature, see, e.g., [49-51,53,54].

Left-right models can have an exact symmetry between left- and right-handed fermions at high scales. One way of doing so is by considering a generalized parity $(P)$ symmetry. In the case of exact $P$ symmetry the QCD $\theta$ term is explicitly forbidden in the microscopic theory. However, there is an explicit $C P$-violating phase $\delta_{2}$ in the Higgs potential,

$$
\alpha_{2}\left\{e^{i \delta_{2}}\left[\operatorname{Tr}\left(\phi \tilde{\phi}^{\dagger}\right) \operatorname{Tr}\left(\Delta_{R} \Delta_{R}^{\dagger}\right)+\operatorname{Tr}\left(\phi^{\dagger} \tilde{\phi}\right) \operatorname{Tr}\left(\Delta_{L} \Delta_{L}^{\dagger}\right)\right]+\text { H.c. }\right\} .
$$

Here $\phi$ is the Higgs bidoublet and $\Delta_{L, R}$ are Higgs triplets. $\delta_{2}$ is responsible for spontaneous $C P$ violation in the Higgs 
bidoublet, $\langle\Phi\rangle=\operatorname{diag}\left(v_{1}, e^{i \alpha} v_{2}\right)$, with $\sin (\alpha) \propto \sin \left(\delta_{2}\right)$ $[55,56]$. Thus the quark mass matrices, and $\bar{\theta}$, obtain a tree-level phase of order $\delta_{2}$, unsuppressed by any ratio of scales.

In left-right symmetric model, $C P$-odd flavor-diagonal operators are induced already at tree level. They arise from the exchange of heavy $W_{R}$ and scalar fields. The contributions from the latter lead to four-fermion operators that scale with SM Yukawa couplings and are thus suppressed for light fermions most relevant for EDMs. In addition, to avoid producing too large flavor-changing neutral currents the scalar fields must be relatively heavy whereas the $W_{R}$ boson could be lighter. We therefore focus on contributions from the tree-level exchange of $W_{R}$ bosons. The tree-level matching leads to a single SMEFT operator right below the mass of the $W_{R}$ boson (see Ref. [54] for a derivation)

$$
\mathcal{L}_{6, \mathrm{MLRSM}}=\frac{c_{H u d}^{i j}}{\Lambda^{2}} i \tilde{\varphi}^{\dagger} D_{\mu} \varphi \bar{u}_{R}^{i} \gamma^{\mu} d_{R}^{j}+\text { H.c. },
$$

where

$$
\frac{c_{H u d}^{i j}}{\Lambda^{2}}=\frac{g_{R}^{2}}{m_{W_{R}}^{2}} \frac{\xi e^{i \alpha}}{1+\xi^{2}} V_{R, i j},
$$

in terms of a gauge coupling $g_{R}=g$, the mass of the righthanded gauge boson $m_{W_{R}}$ and the parameter $\xi$ related to the ratio of VEVs appearing in the model. In this section, we set $V_{R}=V_{\text {CKM }}$ which is a leading-order expression in the limit $\xi \sin \alpha \rightarrow 0$ [57]. Corrections can be systematically included but do not change the qualitative conclusions.
The dimension-six operator $c_{H u d}^{i j}$ appears in Eq. (4) and thus indicates a renormalization of $\bar{\theta}$ at the matching scale. In this case, the EFT argument is conservative as the renormalization already appears at tree level. Nonetheless from a low-energy perspective the appearance of $c_{\mathrm{Hud}}^{i j}$ signifies the need to account for the large $\bar{\theta}$ term.

Below the electroweak scale, after integrating out the electroweak gauge bosons, we obtain four-quark operators

$$
\tilde{\mathcal{L}}_{\text {eff }}=-\sum_{a=1}^{2}\left(C_{a L R}^{i j l m} \mathcal{O}_{a L R}^{i j l m}+C_{a L R}^{i j l m *}\left(\mathcal{O}_{a L R}^{i j l m}\right)^{\dagger}\right),
$$

where

$$
\begin{aligned}
& \mathcal{O}_{1 L R}^{i j l m}=\bar{d}^{m} \gamma^{\mu} P_{L} u^{l} \bar{u}^{i} \gamma_{\mu} P_{R} d^{j}, \\
& \mathcal{O}_{2 L R}^{i j l m}=\bar{d}_{\alpha}^{m} \gamma^{\mu} P_{L} u_{\beta}^{l} \bar{u}_{\beta}^{i} \gamma_{\mu} P_{R} d_{\alpha}^{j},
\end{aligned}
$$

and $\alpha$ and $\beta$ are color indices. At the electroweak scale we have

$$
C_{1 L R}^{i j l m}\left(m_{W}\right)=\frac{V_{l m}^{*} c_{H u d}^{i j}}{\Lambda^{2}}, \quad C_{2 L R}^{i j l m}\left(m_{W}\right)=0
$$

The $C P$-odd four-quark operators depend on the same phase as the induced correction to $\bar{\theta}$.

We focus on operators involving just up, down, and strange quarks and find four relevant operators

$$
\begin{aligned}
\mathcal{L}_{\mathrm{EDM}}= & -i\left(\operatorname{Im} C_{1 L R}^{u d u d} \bar{d} \gamma^{\mu} P_{L} u \bar{u} \gamma_{\mu} P_{R} d+\operatorname{Im} C_{2 L R}^{u d u d} \bar{d}_{\alpha} \gamma^{\mu} P_{L} u_{\beta} \bar{u}_{\beta} \gamma_{\mu} P_{R} d_{\alpha}\right. \\
& \left.+\operatorname{Im} C_{1 L R}^{u s u s} \bar{s} \gamma^{\mu} P_{L} u \bar{u} \gamma_{\mu} P_{R} s+\operatorname{Im} C_{2 L R}^{u s u s} \bar{s}_{\alpha} \gamma^{\mu} P_{L} u_{\beta} \bar{u}_{\beta} \gamma_{\mu} P_{R} s_{\alpha}-\text { H.c. }\right),
\end{aligned}
$$

where the second operator is induced through QCD renormalization-group evolution $[58,59]$

$$
C_{1 L R}^{i j l m}(3 \mathrm{GeV})=0.9 C_{1 L R}^{i j l m}\left(m_{W}\right), \quad C_{2 L R}^{i j l m}(3 \mathrm{GeV})=0.4 C_{1 L R}^{i j l m}\left(m_{W}\right)+1.9 C_{2 L R}^{i j l m}\left(m_{W}\right)
$$

\section{EDM PHENOMENOLOGY}

\section{A. $\boldsymbol{C P}$-violating hadronic couplings}

As discussed above, models with $C P$-violating couplings to the strong sector typically induce a large $\bar{\theta}$ term. For consistency with the neutron EDM the $\bar{\theta}$ term must be relaxed further in the infrared, presumably by a Peccei-Quinn mechanism. The pattern of higher-dimension $C P$-violating operators generated alongside $\bar{\theta}$ in each of the models survives, producing distinctive patterns of electric dipole moments at lower energies. We now turn to the estimation of these EDM observables, a somewhat complicated task that involves hadronic, nuclear, atomic, and molecular physics. In the spirit of effective field theories, it is useful to perform the calculation in steps. We first discuss what hadronic or semileptonic $C P$-violating operators are generated from the various $C P$-violating operators at the quark-gluon level. In principle, many hadronic interactions are generated, but they can be organized in a systematic way in chiral perturbation theory. In this section, we give the main results and refer to Refs. $[60,61]$ for more details.

Semileptonic operators.-We begin with the semileptonic operators that describe $C P$-violating electron-quark 
and electron-gluon interactions. These operators are induced in the leptoquark model, cf. Eqs. (10) and (12). They further induce $C P$-odd electron-nucleon interactions that, in turn, induce atomic and molecular EDMs. The $C P$-violating electron-nucleon interactions take the form [43]

$$
\begin{aligned}
\mathcal{L}= & -\frac{G_{F}}{\sqrt{2}}\left\{\bar{e} i \gamma_{5} e \bar{N}\left(C_{S}^{(0)}+\tau_{3} C_{S}^{(1)}\right) N+\bar{e} e \frac{\partial_{\mu}}{m_{N}}\left[\bar{N}\left(C_{P}^{(0)}+\tau_{3} C_{P}^{(1)}\right) S^{\mu} N\right]\right. \\
& \left.-4 \bar{e} \sigma_{\mu \nu} e \bar{N}\left(C_{T}^{(0)}+\tau_{3} C_{T}^{(1)}\right) v^{\mu} S^{\nu} N\right\}+\ldots
\end{aligned}
$$

where $N=(p n)^{T}$ is the nonrelativistic nucleon doublet with mass $m_{N}$, velocity $v^{\mu}$, and the spin $S^{\mu}\left[v^{\mu}=(1, \mathbf{0})\right.$ and $S^{\mu}=(0, \sigma / 2)$ in the nucleon rest frame]. The matching coefficients are given by

$$
\begin{aligned}
& C_{S}^{(0)}=v^{2}\left[\frac{\sigma_{\pi N}}{m_{u}+m_{d}} \operatorname{Im} C_{\text {lequ }}^{(1) \text { ееии }}+\frac{16 \pi}{9}\left(m_{N}-\sigma_{\pi N}-\sigma_{s}\right) C_{e G}\right], \\
& C_{S}^{(1)}=v^{2} \frac{1}{2} \frac{\delta m_{N}}{m_{d}-m_{u}} \operatorname{Im} C_{\text {lequ }}^{(1) \text { eеии }}, \\
& C_{P}^{(0)}=-8 \pi v^{2}\left(\Delta_{u}+\Delta_{d}\right) m_{N} C_{e \tilde{G}}, \quad C_{P}^{(1)}=v^{2} \frac{g_{A} m_{N}}{m_{u}+m_{d}} \operatorname{Im} C_{\text {lequ }}^{(1) e е u и ~}-8 \pi v^{2} g_{A} m_{N} \frac{m_{d}-m_{u}}{m_{u}+m_{d}} C_{e \tilde{G}}, \\
& C_{T}^{(0)}=v^{2}\left(g_{T}^{d}+g_{T}^{u}\right) \operatorname{Im} C_{\text {lequ }}^{(3) \text { eеии }}, \quad C_{T}^{(1)}=v^{2}\left(g_{T}^{d}-g_{T}^{u}\right) \operatorname{Im} C_{\text {lequ }}^{(3) \text { eеии }} .
\end{aligned}
$$

in terms of the hadronic matrix elements [62-65]

$$
\begin{aligned}
\sigma_{\pi N} & =(59.1 \pm 3.5) \mathrm{MeV}, \quad \sigma_{s}=\left(41.1_{-10.0}^{+11.3}\right) \mathrm{MeV}, \quad \delta m_{N}=(2.32 \pm 0.17) \mathrm{MeV}, \\
g_{A} & =1.27 \pm 0.002, \quad \Delta_{u}=0.842 \pm 0.012, \quad \Delta_{d}=-0.427 \pm 0.013
\end{aligned}
$$

Hadronic operators.-More complicated are the purely hadronic operators such as the quark (chromo-)EDMs and four-quark operators. We begin with the analysis of quark EDMs, which are induced in the LQ scenario as well as the MSSM. Due to the explicit appearance of the electromagnetic field strength, quark EDMs mainly induce hadronic operators that contain explicit photons as well (operators without photons are suppressed by $\left.\alpha_{\mathrm{e} m} / \pi\right)$. The most important operators are the nucleon EDMs, related to the quark EDMs by

$$
\begin{aligned}
& d_{n}\left(d_{q}\right)=g_{T}^{u} d_{u}+g_{T}^{d} d_{d}, \\
& d_{p}\left(d_{q}\right)=g_{T}^{u} d_{d}+g_{T}^{d} d_{u},
\end{aligned}
$$

where $g_{T}^{u}=-0.213 \pm 0.011$ and $g_{T}^{d}=0.820 \pm 0.029$. These so-called tensor charges are obtained from latticeQCD calculations [66] and have very small theoretical uncertainties.

The quark chromo-EDMs also contribute to nucleon EDMs, but there are no lattice-QCD calculations available at present. The neutron EDM was evaluated using QCD sum rules $[67,68]$ giving

$$
d_{n}\left(\tilde{d}_{q}\right)=\tilde{g}_{n}\left(4 Q_{d} \tilde{d}_{d}-Q_{u} \tilde{d}_{u}\right),
$$

where $\tilde{g}_{n}=(1 \pm 0.5) 0.55 e / Q_{u}$. We express the proton EDM through a quark model relation

$$
d_{p}\left(\tilde{d}_{q}\right)=\tilde{c}_{p} \tilde{g}_{n}\left(-4 Q_{d} \tilde{d}_{u}+Q_{u} \tilde{d}_{d}\right)
$$

so that $d_{p}$ and $d_{n}$ depend on the same QCD matrix element $\tilde{g}_{n}$. We use $\tilde{c}_{p}=1 \pm 0.2$ to account for possible isospin breaking. These relations are valid only under a PecceiQuinn mechanism, that is the expressions take into account the contribution from the induced $\bar{\theta}$ term.

In addition to nucleon EDMs, the quark chromo-EDMs also induce $C P$-violating pion-nucleon interactions. The most important operators are given by

$$
L=\bar{g}_{0} \bar{N} \tau \cdot \pi N+\bar{g}_{1} \bar{N} \pi_{3} N
$$

in terms of the pion triplet $\vec{\pi}$. These couplings were evaluated with QCD sum rules as well but come with rather large uncertainties [69]. Chiral perturbation theory can be used to obtain some further insight in these matrix 
elements. Through a chiral transformation it can be shown that the matrix elements connecting $\bar{g}_{0,1}$ to quark chromo-EDMs are related to matrix elements connecting meson and baryon mass corrections to $C P$-even quark chromomagnetic dipole moments [70]. This by itself does not help as the matrix elements of quark chromomagnetic dipole moments are also poorly known. However, Ref. [71] argued that the unknown matrix elements (called the "direct" contributions) are small compared to so-called vacuum-alignment pieces that are much better known. The argument uses a relation between chromomagnetic matrix elements and twist-three distributions that can be measured in deep inelastic scattering processes. Using (sparse) data on the latter, Ref. [71] found that the unknown direct pieces provide only $10 \%$ corrections to the total matrix element. Neglecting the direct pieces, we find the simple relations that are valid in presence of a Peccei-Quinn mechanism,

$$
\bar{g}_{0}\left(\tilde{d}_{q}\right) \simeq \delta_{g_{0}} \frac{1}{4 F_{\pi}}\left(\tilde{d}_{u}+\tilde{d}_{d}\right) r \frac{d \delta m_{N}}{d \bar{m} \varepsilon},
$$

$$
\bar{g}_{1}\left(\tilde{d}_{q}\right) \simeq \delta_{g_{1}} \frac{1}{2 F_{\pi}}\left(\tilde{d}_{u}-\tilde{d}_{d}\right) r \frac{\sigma_{\pi N}}{\bar{m}},
$$

where we defined the vacuum condensate ratio:

$$
r=\frac{1}{2} \frac{\left\langle 0\left|\bar{q} g_{s} \sigma_{\mu \nu} G^{\mu \nu} q\right| 0\right\rangle}{\langle 0|\bar{q} q| 0\rangle}
$$

which has the value $r=(0.4 \pm 0.05) \mathrm{GeV}^{2}$ [72-74]. Furthermore $\quad\left(d \delta m_{N} / d \bar{m} \epsilon\right) \simeq \delta m_{N} /(\bar{m} \epsilon)=(2.49 \pm$ $0.17 \mathrm{MeV}) /(\bar{m} \epsilon)$ [65,75], $\bar{m}=\left(m_{u}+m_{d}\right) / 2=(3.37 \pm$ 0.08) $\mathrm{MeV}$ [76], and $\epsilon=\left(m_{d}-m_{u}\right) /(2 \bar{m})=(0.37 \pm$ $0.03)$ [76]. We have added $\delta_{g_{0,1}}=(1 \pm 0.3)$ to account for the theoretical uncertainties in these expressions.

We now turn to four-quark operators induced, for example, in the MLRSM, cf. Eqs. (33)-(37). We closely follow Ref. [77] which connected the $C P$-odd pion-nucleon couplings through chiral symmetry relations to $K \rightarrow \pi \pi$ amplitudes for which lattice-QCD calculations exist. Put together the relevant expressions are given by

$$
\begin{aligned}
& \bar{g}_{1}=(1.45 \pm 0.16 \pm 0.75) \times 10^{-5} \operatorname{Im}\left(V_{u s}^{*} \frac{v^{2} c_{H u d}^{u s}}{\Lambda^{2}}\right)+(2.85 \pm 0.33 \pm 1.5) \times 10^{-5} \operatorname{Im}\left(V_{u d}^{*} \frac{v^{2} c_{H u d}^{u d}}{\Lambda^{2}}\right), \\
& \bar{g}_{0}=(0.08 \pm 0.015 \pm 0.04) \times 10^{-5} \operatorname{Im}\left(V_{u s}^{*} \frac{v^{2} c_{H u d}^{u s}}{\Lambda^{2}}\right) .
\end{aligned}
$$

The contributions to the nucleon EDMs are not so well understood and have been calculated with several approaches, see, e.g., Refs. [56,78]. We follow Ref. [77] and use the next-to-next-to-leading-order expressions for the chiral loops in dimensional regularization [79]

$$
\begin{aligned}
& d_{n}=\bar{d}_{n}(\mu)-\frac{e g_{A} \bar{g}_{1}}{8 \pi^{2} F_{\pi}}\left(\frac{\bar{g}_{0}}{\bar{g}_{1}}\left(\log \frac{m_{\pi}^{2}}{\mu^{2}}-\frac{\pi m_{\pi}}{2 m_{N}}\right)+\frac{1}{4}\left(\kappa_{1}-\kappa_{0}\right) \frac{m_{\pi}^{2}}{m_{N}^{2}} \log \frac{m_{\pi}^{2}}{\mu^{2}}\right), \\
& d_{p}=\bar{d}_{p}(\mu)+\frac{e g_{A} \bar{g}_{1}}{8 \pi^{2} F_{\pi}}\left(\frac{\bar{g}_{0}}{\bar{g}_{1}}\left(\log \frac{m_{\pi}^{2}}{\mu^{2}}-\frac{2 \pi m_{\pi}}{m_{N}}\right)-\frac{1}{4}\left(\frac{2 \pi m_{\pi}}{m_{N}}+\left(\frac{5}{2}+\kappa_{1}+\kappa_{0}\right) \frac{m_{\pi}^{2}}{m_{N}^{2}} \log \frac{m_{\pi}^{2}}{\mu^{2}}\right)\right),
\end{aligned}
$$

where $g_{A} \simeq 1.27$ is the nucleon axial charge, and $\kappa_{1}=3.7$ and $\kappa_{0}=-0.12$ are related to the nucleon magnetic moments. We set $\mu=m_{N}$ for the renormalization scale and apply $\bar{d}_{n, p}\left(\mu=m_{N}\right)=0$. To estimate the uncertainty of this expression we vary the renormalization scale $\mu$ between $m_{N}$ and $m_{K}$ in the loop expressions. All expressions for the four-quark operators assume a Peccei-Quinn mechanism and include corrections from the induced $\bar{\theta}$ term.

\section{B. The QCD $\overline{\boldsymbol{\theta}}$ term}

If a pattern of EDMs is observed that can be explained by (small) $\bar{\theta}$ term alone, it will not be possible to say whether it is the result of an imperfect infrared relaxation of $\bar{\theta}$ or a small radiative correction to a vanishing ultraviolet boundary condition. This means we can only draw useful conclusions about the strong $C P$ problem if the observable pattern of EDMs is distinguishable from that of a pure $\bar{\theta}$ term. Thus we must compare EDMs induced in the various beyond the SM models discussed above, to those induced by the $\bar{\theta}$ term alone.

Let us briefly discuss the hadronic couplings arising from the $\bar{\theta}$ term. Various lattice-QCD calculations of nucleon EDMs have been reported [2,80-83], and the most accurate result was given in Ref. [2]:

$$
d_{n}=-(1.5 \pm 0.7) \times 10^{-3} \bar{\theta} e \mathrm{fm} .
$$

Other recent lattice calculations found EDMs consistent with zero and thus did not confirm these findings $[82,83]$. 
Equation (51) is in agreement with estimates from QCD sum rules and chiral perturbation theory. The proton EDM was calculated on the lattice as well but the result has a larger uncertainty. Instead we apply a relation $d_{p}=-(1 \pm$ $0.5) d_{n}$ which covers various estimates from the chiral perturbation theory, QCD sum rules, and the lattice results.

The $C P$-odd pion-nucleon couplings are better under control. Reference [84] found

$$
\begin{aligned}
& \bar{g}_{0}=-(14.7 \pm 2.3) \times 10^{-3} \bar{\theta}, \\
& \bar{g}_{1}=(3.4 \pm 2.4) \times 10^{-3} \bar{\theta},
\end{aligned}
$$

through chiral symmetry relations between $C P$-odd pionnucleon interactions and quark-mass corrections to baryon masses.

Finally, Ref. [85] noticed that a nonzero $\bar{\theta}$ term induced $C P$-odd electron-nucleon interactions through electromagnetic loops. They obtained ${ }^{2}$

$$
C_{S}=-(3 \pm 1.5) \times 10^{-2} \bar{\theta},
$$

where $C_{S}$ is a linear combination of $C_{S}^{(0)}$ and $C_{S}^{(1)}$ appearing in Eq. (39). We will discuss this combination in more detail in the next section.

\section{EDMs of nuclei, atoms, and molecules}

We are now in the position to calculate EDMs of various systems. The nucleon EDMs have already been discussed above so we turn to larger systems. We begin with paramagnetic systems that are mainly sensitive to (semi) leptonic $C P$-violating operators. The most stringent limits are from polar molecules due to huge innermolecular electric fields induced by relatively small external electric fields. A nonzero electron EDM and/or electron-nucleon interactions affect the frequency associated with the response of paramagnetic polar molecules to such an applied external field. The frequency $\omega$ is given by

$$
\omega=\alpha_{d_{e}} d_{e}+\alpha_{C_{S}} C_{S},
$$

where the coefficient $C_{S}$ is given by
TABLE II. Input parameters for EDMs of paramagnetic molecules.

\begin{tabular}{lcc}
\hline \hline & $\alpha_{d_{e}}$ & $\alpha_{C_{S}}$ \\
\hline ThO & $\frac{(120.6 \pm 4.9) \mathrm{mrad} / \mathrm{s}}{10^{-27} e \mathrm{~cm}}$ & $(181.6 \pm 7.3) \times 10^{7} \mathrm{mrad} / \mathrm{s}$ \\
$\mathrm{HfF}^{+}$ & $\frac{(34.9 \pm 1.4) \mathrm{mrad} / \mathrm{s}}{10^{-27} e \mathrm{~cm}}$ & $(32.0 \pm 1.3) \times 10^{7} \mathrm{mrad} / \mathrm{s}$ \\
$\mathrm{BaF}$ & $\frac{(19.7 \pm 0.75) \mathrm{mrad} / \mathrm{s}}{10^{-27} e \mathrm{~cm}}$ & $(12.7 \pm 0.18) \times 10^{7} \mathrm{mrad} / \mathrm{s}$ \\
\hline \hline
\end{tabular}

$$
C_{S} \equiv C_{S}^{(0)}+\frac{Z-N}{Z+N} C_{S}^{(1)} .
$$

Here, $Z$ and $N$ are the proton and neutron numbers of the heaviest atom of the molecule. The parameters $\alpha_{d_{e}}$ and $\alpha_{C_{S}}$ depend on the paramagnetic molecular system of interest. As an example, Table II presents the values of $\alpha_{d_{e}}$ and $\alpha_{C_{S}}$

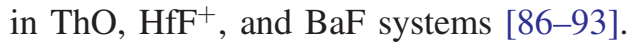

EDMs of nuclei are only sensitive to hadronic sources of $C P$ violation. So far no nuclear EDMs have been measured, but there are plans to measure the EDMs of light nuclei in storage rings [94]. Anticipating such measurements we consider the deuteron EDM [95-97]

$d_{D}=(0.94 \pm 0.01)\left(d_{n}+d_{p}\right)+\left[(0.18 \pm 0.02) \bar{g}_{1}\right] e \mathrm{fm}$.

Diamagnetic atomic EDMs are sensitive to nuclear $C P$ violation and electron-nucleon interactions. ${ }^{225} \mathrm{Ra}$, due to its octopole deformation, is mainly sensitive to $C P$-odd nuclear forces that are, in turn, dominated by one-pionexchange processes involving $\bar{g}_{0,1}$. We use $[98,99]$

$d_{\mathrm{Ra}}=\left(7.7 \times 10^{-4}\right) \times\left[(2.5 \pm 7.5) \bar{g}_{0}-(65 \pm 40) \bar{g}_{1}\right] e$ fm.

The situation is more complicated for the diamagnetic atom ${ }^{199} \mathrm{Hg}$. This system gets relevant contributions from the nucleon EDMs, the $C P$-violating nuclear force, the electron $\mathrm{EDM}$, and from $C P$-odd electron-nucleon interactions. We write [98,100-103]

$$
\begin{aligned}
d_{\mathrm{Hg}}= & (0.012 \pm 0.012) d_{e}-\left[(0.028 \pm 0.006) C_{S}-\frac{1}{3}(3.6 \pm 0.4)\left(C_{T}+\frac{Z \alpha}{5 m_{N} R} C_{P}\right)\right] \times 10^{-20} e \mathrm{~cm} \\
& -(2.1 \pm 0.5) \times 10^{-4}\left[(1.9 \pm 0.1) d_{n}+(0.20 \pm 0.06) d_{p}+\left(0.13_{-0.07}^{+0.5} \bar{g}_{0}+0.25_{-0.63}^{+0.89} \bar{g}_{1}\right) e \mathrm{fm}\right]
\end{aligned}
$$

\footnotetext{
${ }^{2}$ We expect that the same electromagnetic two-loop diagrams discussed in Ref. [85] lead to contributions to $C_{P}$ and $C_{T}$ of similar size. However, these interactions mainly contribute to diamagnetic systems where the $C P$ violation is dominated by purely hadronic interactions. As such, we do not include corrections from $\bar{\theta}$ to $C_{P, T}$ in our analysis.
}

where $R \simeq 1.2 A^{1 / 3} \mathrm{fm}$ is the nuclear radius, and $C_{P, T}=$ $\left(C_{P, T}^{(n)}\left\langle\vec{\sigma}_{n}\right\rangle+C_{P, T}^{(p)}\left\langle\vec{\sigma}_{p}\right\rangle\right) /\left(\left\langle\vec{\sigma}_{n}\right\rangle+\left\langle\vec{\sigma}_{p}\right\rangle\right)$, with $\quad C_{P, T}^{(n, p)}=$ $C_{P, T}^{(0)} \mp C_{P, T}^{(1)}$. For ${ }^{199} \mathrm{Hg}$ we have [104]

$$
\left\langle\vec{\sigma}_{n}\right\rangle=-0.3249 \pm 0.0515, \quad\left\langle\vec{\sigma}_{p}\right\rangle=0.0031 \pm 0.0118 .
$$



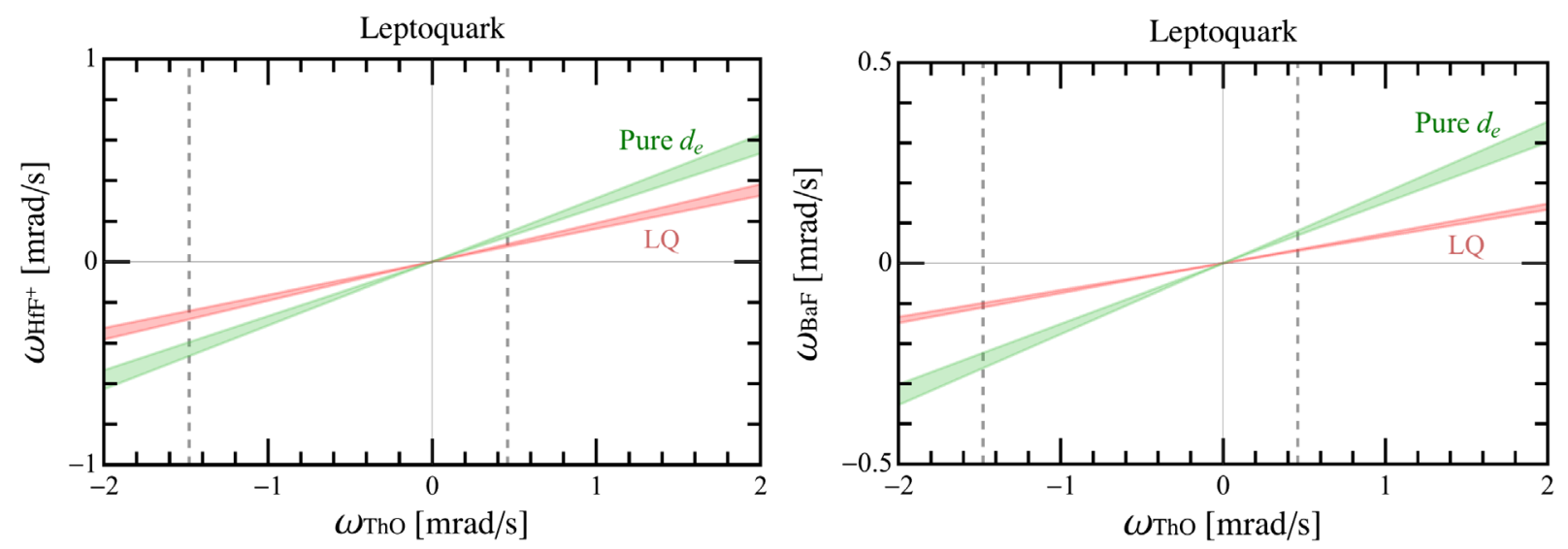

FIG. 1. The left (right) panel shows the correlation between $\omega_{\mathrm{HfF}^{+}}\left(\omega_{\mathrm{BaF}}\right)$ and $\omega_{\mathrm{ThO}}$ in a pure- $d_{e}$ scenario (green) and the LQ scenario (red). The bands indicate the small uncertainty arising from hadronic and molecular matrix elements. The region between the vertical dashed lines is allowed at $2 \sigma$ level.

\section{DISENTANGLING SOURCES OF CP VIOLATION}

In this section we discuss how EDM measurements of various systems can be used to separate various $C P$ violating BSM models and the SMEFT operators they generate from a pure $\bar{\theta}$ scenario. The former, we have argued, require an infrared relaxation of $\bar{\theta}$. The latter, however, is not necessarily indicative of a PQ mechanism, since it could be the remnant of an imperfect UV solution, for instance due to spontaneous breaking of $P$ or $C P$. In addition, as we will discuss, we also have to distinguish scenarios where $C P$ violation is dominated by dimensionsix operators that are not necessarily correlated with large threshold corrections to $\bar{\theta}$. In the models we study the prime example is an electron EDM, which does not appear in Eq. (4).

\section{A. Case 1: Scalar leptoquark models}

We begin with the simple LQ scenario discussed in Sec. II B and focus on couplings between first-generation quarks and leptons. That is, we set $x_{L R}^{e u}=x_{R L}^{u e}=e^{i \alpha}$, where $\alpha$ is some nonzero phase, and set the remaining couplings to zero. In this simple case, the dominant $C P$-violating dimension-six operators are quark-electron interactions, while the electron EDM is one-loop suppressed, which is not compensated by the slight enhancement by $m_{u} / m_{e}$. The quark EDMs and chromo-EDMs also appear only at one loop and are further suppressed by a factor of $m_{e} / m_{u}$.

This scenario precisely predicts ratios between EDMs of paramagnetic systems [102,105]. This is illustrated in Fig. 1 where the red bands in the left and right panels illustrate the ratios $\omega_{\mathrm{HfF}^{+}} / \omega_{\mathrm{ThO}}$ and $\omega_{\mathrm{BaF}} / \omega_{\mathrm{ThO}}$, respectively. The error bands are small as both the hadronic matrix elements connecting electron-quark to electron-nucleon couplings as well as the molecular theory is well under control. The contributions from the electron EDM are very small and appear at the $10^{-4}$ level. Therefore, we can apply the present situation to a sole-source limit in which the bound on $C_{S}$ is obtained by assuming $d_{e}=0$. Taking the current limit, $\left|C_{S}\right|<7.3 \times 10^{-10}$ (90\% C.L.) [106], we obtain $m_{R_{2}} \gtrsim 1.9 \times 10^{4} \mathrm{TeV}$ when $\left|x_{R L}^{e u} x_{R L}^{u e}\right| \sin \alpha=1$, illustrating the excellent sensitivity of EDM experiments to new sources of $C P$ violation.

It is possible to separate this scenario from one where the dominant source of $C P$ violation is the electron EDM. This is, for instance, the case in a LQ model where $x_{L R}^{e t}=x_{R L}^{t e}=$ $e^{i \alpha}$ with the other couplings set to zero. In this case the electron EDM in Eq. (15) is enhanced by $m_{t} / m_{e}$ and provides the dominant contribution to paramagnetic systems. The induced electron-gluon operators provide negligible contributions. In this case, the renormalization of $\bar{\theta}$ is not excessive, as expected from the EFT arguments of Ref. [18], and thus a $d_{e}$-dominated EDM pattern would not necessarily point towards an IR solution of the strong $C P$ problem. A $d_{e}$-dominated scenario like this leads to the green bands in Fig. 1. Clearly, sufficiently precise measurements of two paramagnetic systems can separate these scenarios. The current upper limit on $\omega_{\mathrm{HfF}^{+}}$[107] is a few times weaker than that of $\omega_{\text {ThO }}$ [106], but in general if a nonzero signal is found in one system, then the other one should be around the corner. No measurements of $\omega_{\mathrm{BaF}}$ exist, but an experiment is in development [108].

With just paramagnetic systems it is not possible to separate a pure $\bar{\theta}$ scenario from the LQ scenario with couplings to first-generation quarks. In both cases, the paramagnetic observables are dominated by the $C_{S}$ interactions, and the ratios are therefore almost identical. However, in this case the neutron and diamagnetic EDMs provide a way out. In a pure- $\bar{\theta}$ scenario such EDMs are relatively large compared to paramagnetic EDMs, whereas in the LQ scenario hadronic $C P$ violation is loop suppressed. We illustrate this in Fig. 2 where we depict $\omega_{\text {ThO }}$ versus $d_{n}$. The bands for the LQ and pure $\bar{\theta}$ are 


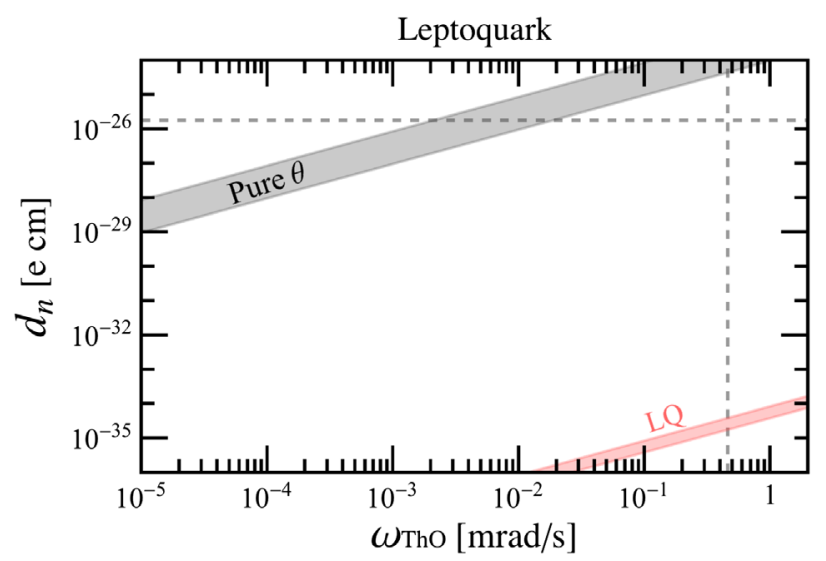

FIG. 2. Correlation between $\omega_{\text {ThO }}$ and $d_{n}$ in the LQ model (red) and a pure- $\bar{\theta}$ scenario (gray). The dashed lines correspond to experimental bounds.

very distinct. For instance, if a next-generation $\mathrm{ThO}$ measurement finds a nonzero value $\omega_{\text {ThO }}=0.1 \mathrm{mrad} / \mathrm{s}$, then a pure $\bar{\theta}$ scenario is already excluded as this would predict $d_{n} \simeq 3 \times 10^{-25} \mathrm{ecm}$, which is well above the existing limit $d_{n} \leq 1.8 \times 10^{-26} \mathrm{e} \mathrm{cm} \mathrm{[1],} \mathrm{depicted} \mathrm{by} \mathrm{the}$ horizontal dashed line. For Hg similar statements hold but here the hadronic and nuclear uncertainties make definite statement more complicated.

In summary, the simple $R_{2}$ leptoquark model illustrates the EFT arguments of [18]. A pattern of EDMs indicative of higher-dimensional $C P$-odd operators that mix quadratically with $\bar{\theta}$ is directly associated with large threshold corrections to $\bar{\theta}$ at the EFT matching scale. The large threshold corrections must be relaxed in the infrared. The leptoquark model also provides an example where paramagnetic EDMs are dominated by a dimension-six operator, the electron EDM, that does not indicate large corrections to $\bar{\theta}$. However, such scenarios can be separated through precise measurements of at least two paramagnetic EDMs.

\section{B. Case II: The MSSM}

Since we have focused on a limiting case of the MSSM where the only phase is in the gluino mass, the EDM phenomenology is dominated by quark EDMs and chromoEDMs. This implies that paramagnetic systems are not particularly relevant for the discussion and we can focus on nucleon, nuclear, and diamagnetic systems. While we have expressed our results in terms of several MSSM parameters, in all ratios of EDMs the dependence on $m_{\text {soft }}$ and $\phi_{3}$ cancels and the only dependence is on the squark mass splitting parametrized by $\Delta$. This dependence is actually mild for $\Delta<1$, where the functions $F(\Delta)$ and $G(\Delta)$ only vary by about $35 \%$, and the ratio of functions $F(\Delta) / G(\Delta)$ only varies by a factor of 2 for any $\Delta$. We assume that an $\mathcal{O}(1)$ splitting among the squarks is a reasonably general region of parameter space and we take $\Delta=1$ for concreteness. Before we discuss ratios of EDMs we briefly mention the reach of present EDM experiments. The current $d_{\mathrm{Hg}}$ limit results in $m_{\mathrm{soft}} \gtrsim 20 \mathrm{TeV}$ at $\phi_{3}=\pi / 2$, taking central values for the hadronic and nuclear matrix elements. The experimental bound on $d_{n}$ leads to the somewhat weaker limit $m_{\text {soft }} \gtrsim 5.4 \mathrm{TeV}$ but has smaller uncertainties.

The neutron and proton EDMs are dominated by the quark chromo-EDMs while the quark EDMs provide roughly $20 \%$ corrections. The ratio of the neutron- and proton-induced chromo-EDM contributions depends on the ratio

$\frac{d_{p}\left(\tilde{d}_{q}\right)}{d_{n}\left(\tilde{d}_{q}\right)}=\tilde{c}_{p} \frac{\left(4 Q_{d} \tilde{d}_{d}-Q_{u} \tilde{d}_{u}\right)}{\left(-4 Q_{d} \tilde{d}_{u}+Q_{u} \tilde{d}_{d}\right)} \simeq-0.78 \times(1 \pm 0.2)$,

where $\tilde{c}_{p}=1 \pm 0.2$ capture the uncertainty due to isospin breaking. Unfortunately, for $\bar{\theta}$-dominated EDMs,

$$
\frac{d_{p}(\bar{\theta})}{d_{n}(\bar{\theta})}=-1 \times(1 \pm 0.5) .
$$

Thus these ratios overlap within uncertainties, implying that measurements of both nucleon EDMs cannot separate a pure $\bar{\theta}$ scenario from our MSSM model. This is clearly illustrated in the top-left panel of Fig. 3, where also EDM contributions are included, where it can be seen that the gray and red bands overlap entirely.

Nuclear and diamagnetic systems are much more promising, as in these cases the contribution from the $C P$-odd nuclear force can break the degeneracy. For instance, in the deuteron EDM the contributions from $d_{n}+d_{p}$ are small in both the MSSM and the pure- $\bar{\theta}$ scenario. However, the contribution from $C P$-odd one-pion-exchange proportional to $\bar{g}_{1}$ is rather different in both models. $\bar{g}_{1}$ is relatively small for $\bar{\theta}$, since the $\bar{\theta}$ term is an isospin-conserving interaction and the generation of $\bar{g}_{1}$ is suppressed by the small quark mass difference over the chiral-symmetrybreaking scale $\Lambda_{\chi} \sim 1 \mathrm{GeV}$. In the MSSM however, the isospin-breaking combination $\tilde{d}_{u}-\tilde{d}_{d}$ induces $\bar{g}_{1}$ directly. As such $\left|d_{D} / d_{n}\right| \gtrsim O(1)$ for the MSSM [54,109] whereas $\left|d_{D} / d_{n}\right| \lesssim O(1)$ for $\bar{\theta}$. This is reflected in the top-right panel of Fig. 3 where, despite sizable hadronic uncertainties, the gray and red bands do not overlap.

A deuteron EDM measurement would be ideal for our goals as it is theoretically relatively clean. However, there is no competitive measurement planned on short timescales, although plans exist for storage-ring experiments. We therefore consider EDMs of diamagnetic atoms. We begin with ${ }^{225} \mathrm{Ra}$, which is analogous to the deuteron EDM as it is dominated by contributions from the isospinbreaking $C P$-violating nuclear force proportional to $\bar{g}_{1}$. Unfortunately, unlike for the deuteron EDM, the nuclear 

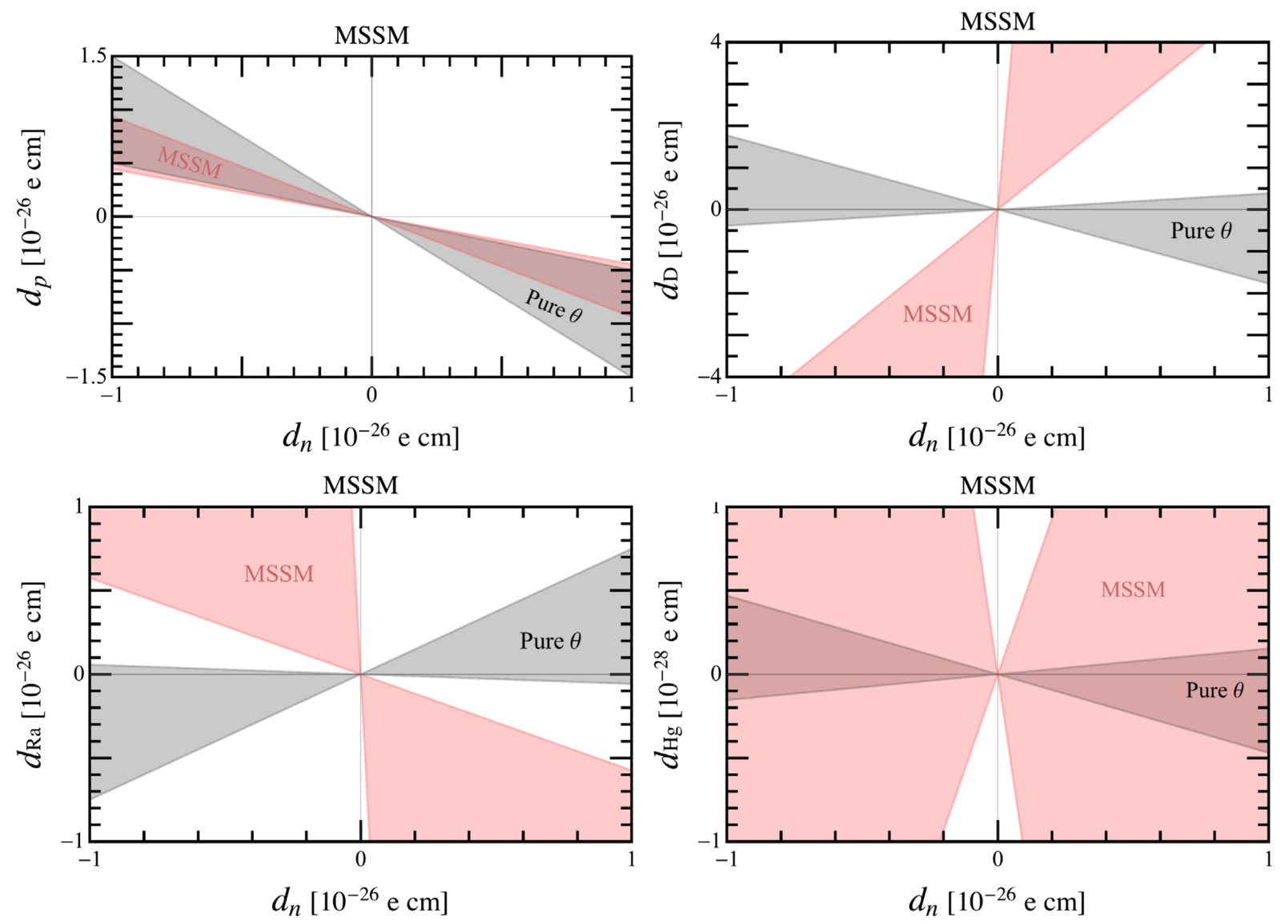

FIG. 3. Correlation between various EDMs and the neutron EDM for a pure- $\bar{\theta}$ scenario (gray) and the MSSM (red). The bands indicate the uncertainty in the ratios arising from hadronic and nuclear matrix elements.

uncertainties are sizable. This is reflected in the bottom-left panel of Fig. 3 where the red and gray bands are wider than was the case for the deuteron. Still, they may be separable. On the other hand, an upper bound on the ${ }^{225} \mathrm{Ra}$ EDM has already been set: $d_{\mathrm{Ra}}<1.2 \times 10^{-23} \mathrm{ecm}$, and a measurement with sensitivity at the $10^{-28} e \mathrm{~cm}$ level is foreseen [110]. If such an experiment sees a signal, the MSSM scenario would predict a somewhat larger neutron EDM than in the pure $\bar{\theta}$ scenario. Slight improvements of nuclear matrix elements would reduce the width of the MSSM bands and make the statements more definite.

Competitive measurements of $d_{D}$ and $d_{\mathrm{Ra}}$ are still in the future, and the most stringent limit is that on $\left|d_{\mathrm{Hg}}\right| \leq$ $6.3 \times 10^{-30} e \mathrm{~cm}$ [111]. Unfortunately, the nuclear uncertainties are severe and make it hard to separate the MSSM from the pure- $\bar{\theta}$ scenario using $d_{\mathrm{Hg}}$. This is illustrated in the bottom-right panel of Fig. 3 where the red and gray bands overlap. Improved calculations of the nuclear matrix elements would go along way in making the predictions more robust. In any case, despite large uncertainties, the pure- $\bar{\theta}$ scenarios can be excluded if measurements fall outside the gray bands. Such a result would be sufficient to make the point.

\section{Case III: The $P$-symmetric minimal left-right-symmetric model}

In the MLRSM the dominant contribution to EDMs arises at tree level from the $C P$-violating four-quark operators in Eq. (37). While most literature focuses on the neutron EDM [56,78,79], nuclear and diamagnetic atoms are actually larger due to the large contribution to $\bar{g}_{1}$ and to lesser extent $\bar{g}_{0}$ in Eq. (49). It was already pointed out in Refs. [54,60] that this leads to enhanced nuclear EDMs, e.g., $\left|d_{D}\right| \gg\left|d_{n}\right|$, and that several measurements can separate contributions from $\bar{\theta}$ and the four-quark operators [54,112]. This can already be glimpsed from the different sensitivities of $d_{n}$ and $d_{\mathrm{Hg}}$ to the left-right symmetry-breaking scale. We $\operatorname{set}^{3} \xi \sin \alpha \simeq m_{b} / m_{t}$, and using central values of the hadronic and nuclear matrix elements obtain $m_{W_{R}} \gtrsim 126(18) \mathrm{TeV}$ from $d_{\mathrm{Hg}}\left(d_{n}\right)$.

We further illustrate this point in Fig. 4. The $d_{p} / d_{n}$ ratio is not well predicted in the MLRSM due to large uncertainties from short-distance contributions not captured by

\footnotetext{
${ }^{3}$ We require $\xi \sin \alpha \leq m_{b} / m_{t}$ to account for the observed quark masses [53].
} 

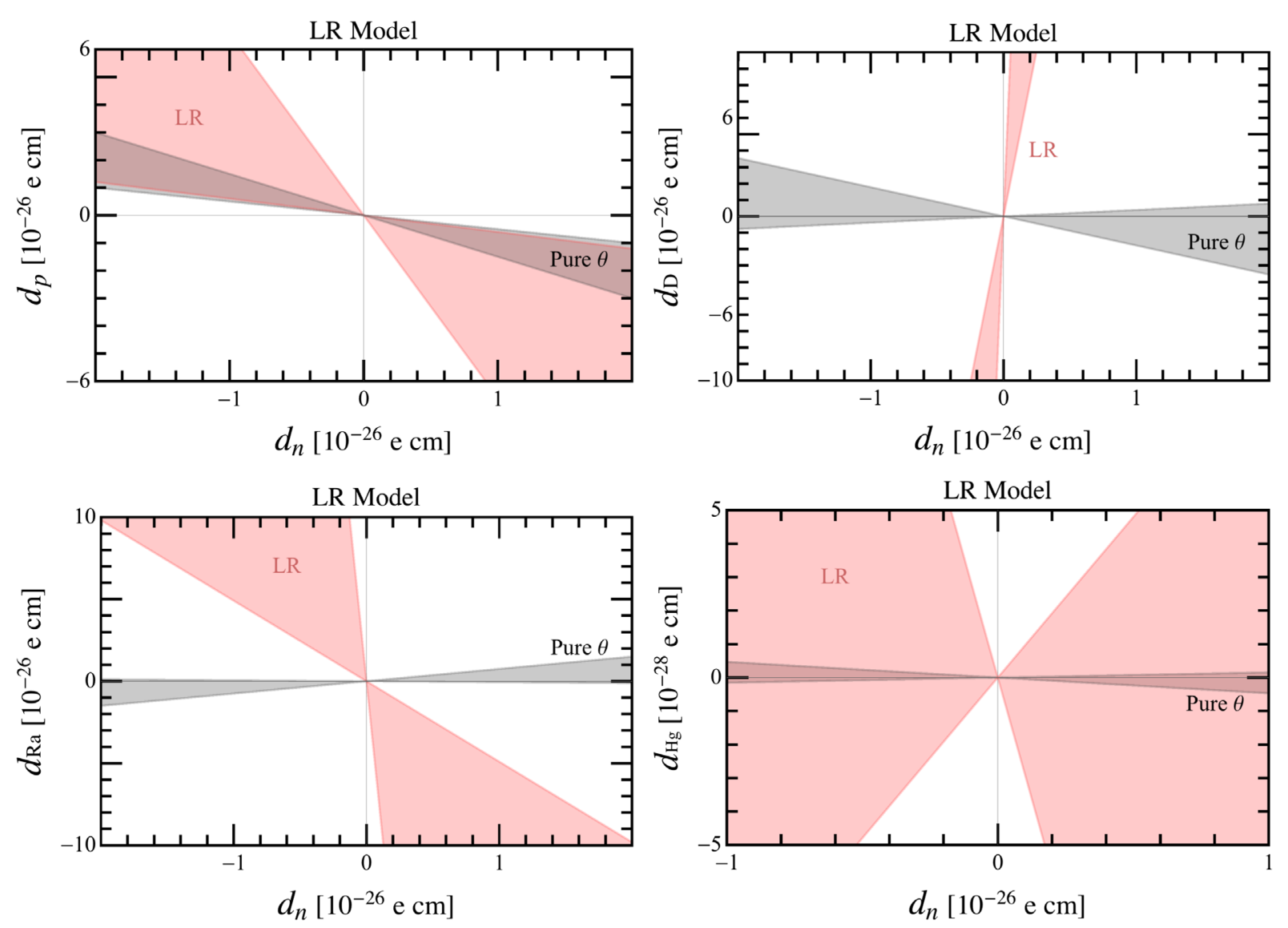

FIG. 4. Correlation between various EDMs and the neutron EDM for a pure- $\bar{\theta}$ scenario (gray) and the MLRSM (red). The bands indicate the uncertainty in the ratios arising from hadronic and nuclear matrix elements.

pion loops. In addition, the MLRSM and pure- $\bar{\theta}$ ratio bands for $d_{p} / d_{n}$ overlap within uncertainties.

The story is completely different for larger systems. The four-quark operators induced in the MLRSM lead to a sizable $C P$-odd nuclear force arising from one-pionexchange diagrams proportional to $\bar{g}_{1}$. Both the hadronic and nuclear matrix elements are well under control leading to a ratio $d_{D} / d_{n}=O(50)$, which is very different from the QCD $\bar{\theta}$ term that predicts much smaller values for the same ratio. The same holds for the diamagnetic atom ${ }^{225} \mathrm{Ra}$, but in this case the nuclear matrix elements are more uncertain, leading to a broader band. Nevertheless, the ratio $d_{\mathrm{Ra}} / d_{n}$ are very distinct in the MLRSM with respect to the pure- $\bar{\theta}$ scenario. In principle, this would hold for $d_{\mathrm{Hg}} / d_{n}$ as well were it not for the large nuclear uncertainties. As a result, the MLRSM and $\bar{\theta}$ bands overlap for their $d_{\mathrm{Hg}} / d_{n}$ predictions.

Finally, let us discuss how a UV solution to the strong $C P$ problem would work in the $P$-symmetric MLRSM. The tree-level correction to $\bar{\theta}$ can be made small by picking a sufficiently small value for the spontaneous phase $\alpha$. In essence this simply transfers the question of the origin of the smallness of $\bar{\theta}$ in the SM, to that of the smallness of $\alpha$.
Nevertheless, some authors have argue this should be considered as a type of solution [113]. In such a UV solution, the $C P$-odd part of the dimension-six operator in Eq. (32) scales with $\alpha$ as well and is suppressed by $v^{2} / v_{R}^{2}$ compared to the tree-level $\bar{\theta}$ term. As such, the low-energy EDM phenomenology is dominated by $\bar{\theta}$ [56]. This is consistent with our assertion that only the presence of dimension-six contributions to EDMs arising from operators in Eq. (4) can be used to infer that strong $C P$ is solved by infrared relaxation.

\section{CONCLUSIONS}

The strong $C P$ problem has been getting worse by about an order of magnitude every decade for the last 60 years [114]. The main experimental probes are the neutron and ${ }^{199} \mathrm{Hg}$ EDMs, which establish the severity of the problem, and searches for axion dark matter. In this work, building on Ref. [18], we have attempted to strengthen the argument that a diverse portfolio of hadronic EDM measurements could provide valuable data on the mechanism that addresses strong $C P$. The basic observation is that it is unnatural to have both new, observably large sources of hadronic $C P$ violation, and an ultraviolet solution to strong 
$C P$. If a pattern of hadronic EDMs is observed that is inconsistent with a small value of $\bar{\theta}$ alone, infrared relaxation by an axion becomes the most natural solution. At the same time, if a Peccei-Quinn mechanism addresses strong $C P$, there is no particular reason for BSM physics to preserve $C P$ to high accuracy.

We have surveyed a handful of BSM models to illustrate this effective field theory argument in detail: when there are large phases, there is a large threshold correction to $\bar{\theta}$ that requires infrared relaxation, and it is correlated with distinctive patterns of hadronic, diamagnetic, and paramagnetic EDMs. These observations motivate targets for future EDM measurements of various systems. In particular, EDM measurements of light nuclei would be very welcome, as the nuclear theory is under good control and the EDMs are not suppressed by Schiff screening. In addition, a rich experimental program is possible with EDMs and magnetic quadrupole moments of radioactive molecules [115] to further constrain hadronic $C P$ violation. To make the most of existing and future experiments, hadronic and nuclear theory must be improved. Lattice QCD calculations are underway that connect dimension-six operators, such as the quark chromo-EDM, to nucleon EDMs $[116,117]$ but still have a long way to go. Improved nuclear structure calculations of, for example, ${ }^{199} \mathrm{Hg}$ and ${ }^{225} \mathrm{Ra}$ EDMs would be very beneficial in connecting observed patterns of EDMs to ultraviolet physics and the nature of the solution to the strong $C P$ problem.

\section{ACKNOWLEDGMENTS}

P. D. acknowledges support from the U.S. Department of Energy under Grant No. DE-SC0015655. K. F. is supported by the U.S. Department of Energy through the Los Alamos National Laboratory and the LANL LDRD Program. Los Alamos National Laboratory is operated by Triad National Security, LLC, for the National Nuclear Security Administration of U.S. Department of Energy (Contract No. 89233218CNA000001). The work of B. L. was performed in part at Aspen Center for Physics, which is supported by National Science Foundation Grant No. PHY1607611. The work of B. L. was partially supported by a grant from the Simons Foundation.
[1] C. Abel et al. (nEDM Collaboration), Phys. Rev. Lett. 124, 081803 (2020).

[2] J. Dragos, T. Luu, A. Shindler, J. de Vries, and A. Yousif, Phys. Rev. C 103, 015202 (2021).

[3] J. R. Ellis and M. K. Gaillard, Nucl. Phys. B150, 141 (1979).

[4] A. E. Nelson, Phys. Lett. 136B, 387 (1984).

[5] S. M. Barr, Phys. Rev. Lett. 53, 329 (1984).

[6] L. Bento, G. C. Branco, and P. A. Parada, Phys. Lett. B 267, 95 (1991).

[7] R. N. Mohapatra and G. Senjanovic, Phys. Lett. 79B, 283 (1978).

[8] M. Beg and H.-S. Tsao, Phys. Rev. Lett. 41, 278 (1978).

[9] H. Georgi, Hadronic J. 1, 155 (1978).

[10] K. S. Babu and R. N. Mohapatra, Phys. Rev. D 41, 1286 (1990).

[11] S. M. Barr, D. Chang, and G. Senjanovic, Phys. Rev. Lett. 67, 2765 (1991).

[12] M. Dine, R. G. Leigh, and A. Kagan, Phys. Rev. D 48, 2214 (1993).

[13] G. Hiller and M. Schmaltz, Phys. Lett. B 514, 263 (2001).

[14] M. Dine and P. Draper, J. High Energy Phys. 08 (2015) 132.

[15] A. Albaid, M. Dine, and P. Draper, J. High Energy Phys. 12 (2015) 046.

[16] P. Draper and D. McKeen, J. High Energy Phys. 04 (2016) 127.

[17] P. Draper, J. Kozaczuk, and J.-H. Yu, Phys. Rev. D 98, 015028 (2018).
[18] J. de Vries, P. Draper, K. Fuyuto, J. Kozaczuk, and D. Sutherland, Phys. Rev. D 99, 015042 (2019),

[19] J. de Vries, H. Patel, and P. Draper, arXiv:2109.01630.

[20] B. Grzadkowski, M. Iskrzynski, M. Misiak, and J. Rosiek, J. High Energy Phys. 10 (2010) 085.

[21] R. D. Peccei and H. R. Quinn, Phys. Rev. D 16, 1791 (1977).

[22] R. D. Peccei and H. R. Quinn, Phys. Rev. Lett. 38, 1440 (1977).

[23] F. Wilczek, Phys. Rev. Lett. 40, 279 (1978).

[24] S. Weinberg, Phys. Rev. Lett. 40, 223 (1978).

[25] J. E. Kim, Phys. Rev. Lett. 43, 103 (1979).

[26] M. A. Shifman, A. I. Vainshtein, and V. I. Zakharov, Nucl. Phys. B166, 493 (1980).

[27] M. Dine, W. Fischler, and M. Srednicki, Phys. Lett. 104B, 199 (1981).

[28] A. R. Zhitnitsky, Yad. Fiz. 31, 497 (1980) [Sov. J. Nucl. Phys. 31, 260 (1980)].

[29] E. J. Chun and A. Lukas, Phys. Lett. B 297, 298 (1992).

[30] L. Randall, Phys. Lett. B 284, 77 (1992).

[31] H.-C. Cheng and D. E. Kaplan, arXiv:hep-ph/0103346.

[32] L. Di Luzio, E. Nardi, and L. Ubaldi, Phys. Rev. Lett. 119, 011801 (2017).

[33] H. Fukuda, M. Ibe, M. Suzuki, and T. T. Yanagida, Phys. Lett. B 771, 327 (2017).

[34] B. Lillard and T. M. P. Tait, J. High Energy Phys. 11 (2017) 005.

[35] B. Lillard and T. M. P. Tait, J. High Energy Phys. 11 (2018) 199. 
[36] G. Choi, M. Suzuki, and T. T. Yanagida, J. High Energy Phys. 07 (2020) 048.

[37] M. Ardu, L. Di Luzio, G. Landini, A. Strumia, D. Teresi, and J.-W. Wang, J. High Energy Phys. 11 (2020) 090.

[38] Y. Nakai and M. Suzuki, Phys. Lett. B 816, 136239 (2021).

[39] L. Darmé and E. Nardi, arXiv:2102.05055.

[40] H.-S. Lee and W. Yin, Phys. Rev. D 99, 015041 (2019).

[41] W. Yin, J. High Energy Phys. 10 (2020) 032.

[42] I. Doršner, S. Fajfer, A. Greljo, J. F. Kamenik, and N. Košnik, Phys. Rep. 641, 1 (2016).

[43] W. Dekens, J. de Vries, M. Jung, and K. K. Vos, J. High Energy Phys. 01 (2019) 069.

[44] K. Fuyuto, M. Ramsey-Musolf, and T. Shen, Phys. Lett. B 788, 52 (2019).

[45] X.-G. He, B. H. J. McKellar, and S. Pakvasa, Phys. Lett. B 283, 348 (1992).

[46] S. M. Barr, Phys. Rev. D 45, 4148 (1992).

[47] J. R. Ellis, J. S. Lee, and A. Pilaftsis, J. High Energy Phys. 10 (2008) 049.

[48] T. Ibrahim and P. Nath, Phys. Rev. D 57, 478 (1998); 58, 019901(E) (1998); 60, 079903(E) (1999); 60, 119901(E) (1999).

[49] J. C. Pati and A. Salam, Phys. Rev. D 10, 275 (1974); 11, 703(E) (1975).

[50] R. N. Mohapatra and J. C. Pati, Phys. Rev. D 11, 566 (1975).

[51] G. Senjanovic and R. N. Mohapatra, Phys. Rev. D 12, 1502 (1975).

[52] W. Dekens and D. Boer, Nucl. Phys. B889, 727 (2014).

[53] A. Maiezza, M. Nemevsek, F. Nesti, and G. Senjanovic, Phys. Rev. D 82, 055022 (2010).

[54] W. Dekens, J. de Vries, J. Bsaisou, W. Bernreuther, C. Hanhart, U.-G. Meißner, A. Nogga, and A. Wirzba, J. High Energy Phys. 07 (2014) 069.

[55] K. Kiers, M. Assis, and A. A. Petrov, Phys. Rev. D 71, 115015 (2005).

[56] A. Maiezza and M. Nemevšek, Phys. Rev. D 90, 095002 (2014).

[57] G. Senjanović and V. Tello, Phys. Rev. Lett. 114, 071801 (2015).

[58] J. Hisano, K. Tsumura, and M. J. S. Yang, Phys. Lett. B 713, 473 (2012).

[59] W. Dekens and J. de Vries, J. High Energy Phys. 05 (2013) 149.

[60] J. de Vries, E. Mereghetti, R. G. E. Timmermans, and U. van Kolck, Ann. Phys. (Amsterdam) 338, 50 (2013).

[61] J. de Vries, E. Epelbaum, L. Girlanda, A. Gnech, E. Mereghetti, and M. Viviani, Front. Phys. 8, 218 (2020).

[62] A. Airapetian et al. (HERMES Collaboration), Phys. Rev. D 75, 012007 (2007).

[63] M. Hoferichter, J. Ruiz de Elvira, B. Kubis, and U.-G. Meißner, Phys. Rev. Lett. 115, 092301 (2015).

[64] A. Abdel-Rehim, C. Alexandrou, M. Constantinou, K. Hadjiyiannakou, K. Jansen, C. Kallidonis, G. Koutsou, and A. Vaquero Aviles-Casco (ETM Collaboration), Phys. Rev. Lett. 116, 252001 (2016).

[65] S. Borsanyi et al., Science 347, 1452 (2015).

[66] R. Gupta, B. Yoon, T. Bhattacharya, V. Cirigliano, Y.-C. Jang, and H.-W. Lin, Phys. Rev. D 98, 091501 (2018).

[67] M. Pospelov and A. Ritz, Phys. Rev. D 63, 073015 (2001).
[68] J. Hisano, J. Y. Lee, N. Nagata, and Y. Shimizu, Phys. Rev. D 85, 114044 (2012).

[69] M. Pospelov, Phys. Lett. B 530, 123 (2002).

[70] J. de Vries, E. Mereghetti, C.-Y. Seng, and A. WalkerLoud, Phys. Lett. B 766, 254 (2017).

[71] C.-Y. Seng, Phys. Rev. Lett. 122, 072001 (2019).

[72] V. Belyaev and I. Ioffe, Sov. Phys. JETP 100, 493 (1982).

[73] V. Belyaev and Y. Kogan, Sov. J. Nucl. Phys. 40, 659 (1984).

[74] S. Narison, Cambridge Monogr. Part. Phys., Nucl. Phys., Cosmol. 17, 1 (2002).

[75] S. Borsanyi et al. (Budapest-Marseille-Wuppertal Collaboration), Phys. Rev. Lett. 111, 252001 (2013).

[76] S. Aoki et al., Eur. Phys. J. C 77, 112 (2017).

[77] V. Cirigliano, W. Dekens, J. de Vries, and E. Mereghetti, Phys. Lett. B 767, 1 (2017).

[78] Y. Zhang, H. An, X. Ji, and R. N. Mohapatra, Nucl. Phys. B802, 247 (2008).

[79] C.-Y. Seng, J. de Vries, E. Mereghetti, H. H. Patel, and M. Ramsey-Musolf, Phys. Lett. B 736, 147 (2014).

[80] F. K. Guo, R. Horsley, U. G. Meissner, Y. Nakamura, H. Perlt, P. E. L. Rakow, G. Schierholz, A. Schiller, and J. M. Zanotti, Phys. Rev. Lett. 115, 062001 (2015).

[81] M. Abramczyk, S. Aoki, T. Blum, T. Izubuchi, H. Ohki, and S. Syritsyn, Phys. Rev. D 96, 014501 (2017).

[82] C. Alexandrou, A. Athenodorou, K. Hadjiyiannakou, and A. Todaro, Phys. Rev. D 103, 054501 (2021).

[83] T. Bhattacharya, V. Cirigliano, R. Gupta, E. Mereghetti, and B. Yoon, Phys. Rev. D 103, 114507 (2021).

[84] J. de Vries, E. Mereghetti, and A. Walker-Loud, Phys. Rev. C 92, 045201 (2015).

[85] V. V. Flambaum, M. Pospelov, A. Ritz, and Y. V. Stadnik, Phys. Rev. D 102, 035001 (2020).

[86] M. Denis and T. Fleig, J. Chem. Phys. 145, 214307 (2016).

[87] L. V. Skripnikov, A. N. Petrov, and A. V. Titov, J. Chem. Phys. 139, 221103 (2013).

[88] T. Fleig, Phys. Rev. A 96, 040502 (2017).

[89] L. V. Skripnikov, J. Chem. Phys. 145, 214301 (2016).

[90] M. Abe, G. Gopakumar, M. Hada, B. P. Das, H. Tatewaki, and D. Mukherjee, Phys. Rev. A 90, 022501 (2014).

[91] A. Sunaga, M. Abe, M. Hada, and B. P. Das, Phys. Rev. A 93, 042507 (2016).

[92] M. Denis, P. A. B. Haase, R. G. E. Timmermans, E. Eliav, N. R. Hutzler, and A. Borschevsky, Phys. Rev. A 99, 042512 (2019).

[93] P. A. B. Haase, D. J. Doeglas, A. Boeschoten, E. Eliav, M. Ilia, P. Aggarwal, H. L. Bethlem, A. Borschevsky, K. Esajas, Y. Hao, S. Hoekstra, V. R. Marshall, T. B. Meijknecht, M. C. Mooij, K. Steinebach, R. G. E. Timmermans, A. Touwen, W. Ubachs, L. Willmann, and Y. Yin, J. Chem. Phys. 155, 034309 (2021).

[94] F. Abusaif et al. (CPEDM Collaboration), arXiv:1912 .07881 .

[95] J. Bsaisou, J. de Vries, C. Hanhart, S. Liebig, U.-G. Meissner, D. Minossi, A. Nogga, and A. Wirzba, J. High Energy Phys. 03 (2015) 104; 05 (2015) 083(E).

[96] N. Yamanaka and E. Hiyama, Phys. Rev. C 91, 054005 (2015). 
[97] A. S. Zhevlakov and V. E. Lyubovitskij, Phys. Rev. D 101, 115041 (2020).

[98] J. Engel, M. J. Ramsey-Musolf, and U. van Kolck, Prog. Part. Nucl. Phys. 71, 21 (2013).

[99] J. Dobaczewski, J. Engel, M. Kortelainen, and P. Becker, Phys. Rev. Lett. 121, 232501 (2018).

[100] V. F. Dmitriev and R. A. Sen'kov, Phys. At. Nucl. 66, 1940 (2003).

[101] N. Yamanaka, B. K. Sahoo, N. Yoshinaga, T. Sato, K. Asahi, and B. P. Das, Eur. Phys. J. A 53, 54 (2017).

[102] T. Fleig and M. Jung, J. High Energy Phys. 07 (2018) 012.

[103] B. K. Sahoo and B. P. Das, Phys. Rev. Lett. 120, 203001 (2018).

[104] K. Yanase, N. Yoshinaga, K. Higashiyama, and N. Yamanaka, Phys. Rev. D 99, 075021 (2019).

[105] T. Chupp and M. Ramsey-Musolf, Phys. Rev. C 91, 035502 (2015).

[106] V. Andreev et al. (ACME Collaboration), Nature (London) 562, 355 (2018).
[107] W. B. Cairncross, D. N. Gresh, M. Grau, K. C. Cossel, T. S. Roussy, Y. Ni, Y. Zhou, J. Ye, and E. A. Cornell, Phys. Rev. Lett. 119, 153001 (2017).

[108] P. Aggarwal et al. (NL-eEDM Collaboration), Eur. Phys. J. D 72, 197 (2018).

[109] O. Lebedev, K. A. Olive, M. Pospelov, and A. Ritz, Phys. Rev. D 70, 016003 (2004).

[110] M. Bishof et al., Phys. Rev. C 94, 025501 (2016).

[111] B. Graner, Y. Chen, E. G. Lindahl, and B. R. Heckel, Phys. Rev. Lett. 116, 161601 (2016).

[112] M. J. Ramsey-Musolf and J. C. Vasquez, Phys. Lett. B 815, 136136 (2021).

[113] G. Senjanovic and V. Tello, arXiv:2004.04036.

[114] J. H. Smith, E. M. Purcell, and N. F. Ramsey, Phys. Rev. 108, 120 (1957).

[115] N. R. Hutzler et al., arXiv:2010.08709.

[116] A. Shindler, Eur. Phys. J. A 57, 128 (2021).

[117] J. Kim, T. Luu, M. D. Rizik, and A. Shindler, arXiv:2106 .07633 . 